\title{
UNIVERSAL ABELIAN VARIETY AND SIEGEL MODULAR FORMS
}

\author{
SHOUHEI MA
}

\begin{abstract}
Aвstract. We prove that the ring of Siegel modular forms of weight divisible by $g+n+1$ is isomorphic to the ring of $(\log )$ pluricanonical forms on the $n$-fold Kuga family of abelian varieties and its certain compactifications, for every arithmetic group for a symplectic form of rank $2 g>2$. We also give applications to the Kodaira dimension of the Kuga variety. In most cases, the Kuga variety has canonical singularities.
\end{abstract}

\section{INTRODUCTION}

Our purpose in this article is to establish a correspondence between Siegel modular forms and pluricanonical forms on the universal family of abelian varieties and its compactification, which connects modular forms to the geometry of the universal family. Let $\Lambda$ be a free $\mathbb{Z}$-module of rank $2 g>2$ equipped with a nondegenerate symplectic form $\Lambda \times \Lambda \rightarrow \mathbb{Z}$, and $\Gamma$ be a finite-index subgroup of the symplectic group $\operatorname{Sp}(\Lambda)$ of $\Lambda$. Let $A(\Gamma)=\mathcal{D} / \Gamma$ be the Siegel modular variety defined by $\Gamma$, where $\mathcal{D}$ is the Hermitian symmetric domain attached to $\Lambda$. Over $A(\Gamma)$ we have the $n$-fold Kuga family $X^{n}(\Gamma) \rightarrow A(\Gamma)$, whose general fibers are $n$-fold self products of the abelian varieties or their quotient by -1 , according to whether $-1 \notin \Gamma$ or $-1 \in \Gamma$. The space $X^{n}(\Gamma)$ is a normal quasi-projective variety of dimension $g(g+2 n+1) / 2$. Let $M_{k}(\Gamma)$ be the space of Siegel modular forms of weight $k$ with respect to $\Gamma$, and $S_{k}(\Gamma)$ be the subspace of cusp forms. Our starting point is the following correspondence.

Theorem 1.1 (\$7). We have a natural isomorphism of graded rings

$$
\bigoplus_{m \geq 0} H^{0}\left(X^{n}(\Gamma), K_{X^{n}(\Gamma)}^{\otimes m}\right) \simeq \bigoplus_{m \geq 0} M_{(g+n+1) m}(\Gamma) .
$$

If $X$ is a smooth projective model of $X^{n}(\Gamma)$, the $m=1$ component of (1.1) induces an isomorphism

$$
H^{0}\left(X, K_{X}\right) \simeq S_{g+n+1}(\Gamma) .
$$

The isomorphism (1.1) is a consequence of natural isomorphisms between the relevant line bundles on $\mathcal{D}$. A higher analogue of (1.1) is given

Supported by JSPS KAKENHI 15H05738 and 17K14158. 
in the form of a Leray spectral sequence that relates vector-valued Siegel modular forms to the cohomology of $K_{X^{n}(\Gamma)}^{\otimes m}(\$ 7.3)$. The isomorphism (1.2) is a direct extension of the result of Hatada [8] who considered the case $\Gamma<\operatorname{Sp}(2 g, \mathbb{Z})$ torsion-free, and is a generalization of the result of Shioda [28] and Shokurov [29] in the case $g=1$.

Our main result is an extension of (1.1) to a certain class of compactification $X$ of $X^{n}(\Gamma)$. Although our principal interest would be in compact $X$, the result also holds for not fully compact $X$ as well.

Theorem $1.2(\$[)$. Let $X$ be a complex analytic variety which contains $X^{n}(\Gamma)$ as a Zariski open set. Assume that

- the singular locus of $X$ has codimension $\geq 2$,

- $X^{n}(\Gamma) \rightarrow A(\Gamma)$ extends to a morphism $X \rightarrow A(\Gamma)^{\Sigma}$ to some toroidal compactification $A(\Gamma)^{\Sigma}$ of $A(\Gamma)$, and

- every irreducible component of the boundary divisor $\Delta_{X}=X-X^{n}(\Gamma)$ of $X$ dominates some irreducible component of the boundary divisor $\Delta_{A}=A(\Gamma)^{\Sigma}-A(\Gamma)$ of $A(\Gamma)^{\Sigma}$.

Then the isomorphism (1.1) extends to an isomorphism

$$
\bigoplus_{m \geq 0} H^{0}\left(X, K_{X}^{\otimes m}\left(m \Delta_{X}\right)\right) \simeq \bigoplus_{m \geq 0} M_{(g+n+1) m}(\Gamma) .
$$

For each $m$, this gives an injection

$$
S_{(g+n+1) m}(\Gamma) \hookrightarrow H^{0}\left(K_{X}^{\otimes m}\left((m-1) \Delta_{X}\right)\right) .
$$

If $X$ is compact and the singularities of the pair $\left(X,\left(1-m^{-1}\right) \Delta_{X}\right)$ is Kawamata log terminal over general points of $\Delta_{A}$, then (1.4) is also surjective.

Namikawa [20] was the first to construct an example of such a compactification $X$ for $n=1$ and $\Gamma$ the principal congruence subgroups of $\operatorname{Sp}(2 g, \mathbb{Z})$ of even level $\geq 4$, where $X$ is nonsingular and is a projective family over the 2nd Voronoi compactification of $A(\Gamma)$. Namikawa constructed his $X$ as a toroidal compactification of $X^{1}(\Gamma)$. General theory of toroidal compactification of $X^{n}(\Gamma)$ has been then developed in [22], [3], [14]. A feature of Theorem 1.2 is that it is obtained without knowing specific geometry of the boundary of $X$.

The isomorphism (1.3) is derived by showing that every $m$-canonical form $\omega$ on $X^{n}(\Gamma)$ has at most pole of order $m$ along every component of $\Delta_{X}$ (a Koecher type statement). We deduce this property by deriving an asymptotic estimate of the $L^{2 / m}$ norm of $\omega$ around $\Delta_{X}$. As a key step, we use the isomorphism (1.1) to translate the $L^{2 / m}$ norm of $\omega$ into the Petersson norm of the corresponding modular form. The problem is then reduced to asymptotic estimate of the Petersson norm of modular forms around $\Delta_{A}$, which is derived by a standard calculation. 
Theorem 1.2 generalizes the result of [16] in the case $g=1$, which in turn is another generalization of the Shioda isomorphism. Several new features arise in the case $g>1$, such as the Koecher principle and toroidal compactification, which make some part of the story different from the case $g=1$.

We also give applications of Theorems 1.1 and 1.2 to the Kodaira dimension $\kappa\left(X^{n}(\Gamma)\right)$ of $X^{n}(\Gamma)$. Note that $\kappa\left(X^{n}(\Gamma)\right)$ is nondecreasing with respect to $n$ ([32]), and is bounded by $g(g+1) / 2$ ([12]). In particular, $\kappa\left(X^{n}(\Gamma)\right)=g(g+1) / 2$ when $A(\Gamma)$ is of general type. In general, the isomorphism (1.2) and knowledge about cusp forms tell us a bound of $n$ for $\kappa\left(X^{n}(\Gamma)\right) \geq 0$ (cf. Example 7.4). Moreover, we have the following.

Theorem $1.3(\$ 9)$. (1) Let $k_{0}$ be a weight such that $S_{k_{0}}(\Gamma)$ gives a generically finite map $A(\Gamma) \rightarrow \mathbb{P}^{N}$. Then $\kappa\left(X^{n}(\Gamma)\right)=g(g+1) / 2$ for $n \geq k_{0}-g-1$.

(2) Let $X \supset X^{n}(\Gamma)$ be a normal compact complex analytic variety which satisfies the conditions in Theorem 1.2. Then we have

$$
\kappa\left(A(\Gamma)^{\Sigma},(g+n+1) L-\Delta_{A}\right) \leq \kappa\left(K_{X}\right) \leq g(g+1) / 2
$$

where $L$ is the $\mathbb{Q}$-line bundle of modular forms of weight 1.

Here, for a $\mathbb{Q}$-divisor $D$ on a normal compact complex analytic variety $X$, we write $\kappa(D)=\kappa(X, D)$ for its litaka dimension. We have $\kappa(X) \leq \kappa\left(K_{X}\right)$, where equality holds if $X$ has canonical singularities.

By $(1), \kappa\left(X^{n}(\Gamma)\right)$ stabilizes to $g(g+1) / 2$ for large $n$. In general, it is not easy to explicitly find a weight $k_{0}$ as in (1), and also the resulting bound $n=k_{0}-g-1$ for $\kappa\left(X^{n}(\Gamma)\right)=g(g+1) / 2$ would be far from the actual bound as we are looking only at the canonical map. This is improved by (2), which reduces the problem to the study of slope of cusps forms (leaving aside the singularities). The behavior of $\kappa\left(k L-\Delta_{A}\right)$ is relatively more tractable. As for the singularities, at least $X^{n}(\Gamma)$ has canonical singularities in most cases (\$10).

This paper is organized as follows. $\$ 2-\$ 4$ are recollection of Siegel modular varieties and toroidal compactification. In $\$ 5$ we prepare an asymptotic estimate of the Petersson norm of local modular forms. In $\$ 6$ we prepare a general $L^{2 / m}$ criterion for log pluricanonical forms. In \$7, which can be read after $\$ 2$, we prove Theorem 1.1 . In $\$ 8$ we prove Theorem 1.2 , In $\$ 9$ we prove Theorem 1.3 . In $\$ 10$, which is independent of other sections, we prove that $X^{n}(\Gamma)$ has canonical singularities in most cases.

I wish to thank Gavril Farkas for valuable comments which led me to study Theorem 1.3 . 


\section{Preliminaries}

In this section we recall Siegel modular variety, Siegel modular forms, and Kuga family.

2.1. Universal marked family. Let $\Lambda$ be a free abelian group of rank $2 g>$ 2 endowed with a nondegenerate symplectic form $(\cdot, \cdot): \Lambda \times \Lambda \rightarrow \mathbb{Z}$. Let $\operatorname{LG}\left(\Lambda_{\mathbb{C}}\right)=\operatorname{LG}\left(g, \Lambda_{\mathbb{C}}\right)$ be the Lagrangian Grassmannian parametrizing $g$ dimensional (= maximal) isotropic subspaces. The Hermitian symmetric domain attached to $\Lambda$ is the open subset of $\operatorname{LG}\left(\Lambda_{\mathbb{C}}\right)$ defined by

$$
\mathcal{D}=\left\{[V] \in \operatorname{LG}\left(\Lambda_{\mathbb{C}}\right)|i(\cdot, \cdot)|_{V}>0\right\} .
$$

Here $\left.i(\cdot, \bar{r})\right|_{V}>0$ means the condition that the Hermitian form $\left.i(\cdot, \bar{\bullet})\right|_{V}$ on $V$ is positive definite. This ensures that $\Lambda_{\mathbb{C}}=V \oplus \bar{V}$ for $[V] \in \mathcal{D}$.

Let $\Lambda_{\mathbb{C}}=\mathcal{D} \times \Lambda_{\mathbb{C}}$ be the product vector bundle over $\mathcal{D}, E \rightarrow \mathcal{D}$ the universal sub bundle of $\Lambda_{\mathbb{C}}$ whose fiber over $[V] \in \mathcal{D}$ is $V \subset \Lambda_{\mathbb{C}}$, and $F=\underline{\Lambda_{\mathbb{C}}} / E$ the universal quotient bundle. The symplectic pairing defines a canonical isomorphism $F \simeq E^{\vee}$. The tangent bundle of $\mathcal{D}$ is canonically isomorphic to $\operatorname{Sym}^{2} F$.

The local system $\underline{\Lambda}=\mathcal{D} \times \Lambda$ inside $\Lambda_{\mathbb{C}}$ induces a local system of sections of $F \rightarrow \mathcal{D}$. The universal family of abelian varieties over $\mathcal{D}$ is defined by

$$
\mathcal{X}=F / \underline{\Lambda}=\underline{\Lambda_{\mathbb{C}}} /(E+\underline{\Lambda}) \simeq E^{\vee} / \underline{\Lambda} .
$$

Here the last isomorphism is defined by the symplectic pairing. The fiber of the projection $f: \mathcal{X} \rightarrow \mathcal{D}$ over $[V] \in \mathcal{D}$ is the abelian variety

$$
A=\Lambda_{\mathbb{C}} /(V+\Lambda) \simeq V^{\vee} / \Lambda,
$$

polarized by the symplectic form on $\Lambda \simeq H_{1}(A, \mathbb{Z})$. We can naturally identify $H^{0}\left(\Omega_{A}^{1}\right)=V$. Hence if $\Omega_{f}^{1}$ is the relative cotangent bundle of $f$, we have a canonical isomorphism $f_{*} \Omega_{f}^{1} \simeq E$.

Let $L=\operatorname{det} E$. This is restriction of the tautological line bundle $O(-1)$ over $\mathbb{P}\left(\bigwedge^{g} \Lambda_{\mathbb{C}}\right)$ by the Plücker embedding $\mathcal{D} \subset \operatorname{LG}\left(\Lambda_{\mathbb{C}}\right) \hookrightarrow \mathbb{P}\left(\bigwedge^{g} \Lambda_{\mathbb{C}}\right)$. The fiber of $L$ over $[V] \in \mathcal{D}$ is $\operatorname{det} V=H^{0}\left(K_{A}\right)$. Hence if $K_{f}=\operatorname{det} \Omega_{f}^{1}$ is the relative canonical bundle of $f$, we have a natural isomorphism $f_{*} K_{f} \simeq$ $L$. Since $\left.K_{f}\right|_{A} \simeq O_{A}$ for every fiber $A$ of $f$, the natural homomorphism $f^{*} f_{*} K_{f} \rightarrow K_{f}$ is isomorphic. Therefore $K_{f} \simeq f^{*} L$. On the other hand, taking determinant of $\Omega_{\mathcal{D}}^{1} \simeq \operatorname{Sym}^{2} E$, we also have an $\operatorname{Sp}\left(\Lambda_{\mathbb{R}}\right)$-equivariant isomorphism

$$
K_{\mathcal{D}} \simeq \operatorname{det}\left(\operatorname{Sym}^{2} E\right) \simeq L^{\otimes g+1}
$$

For a natural number $n$ we take the $n$-fold self fiber product

$$
\mathcal{X}^{(n)}=\mathcal{X} \times_{\mathcal{D}} \cdots \times_{\mathcal{D}} \mathcal{X} \simeq F^{\oplus n} / \underline{\Lambda}^{\oplus n}
$$


and let $f_{n}: \mathcal{X}^{(n)} \rightarrow \mathcal{D}$ be the projection. Since $L_{[V]}^{\otimes n} \simeq H^{0}\left(K_{A}\right)^{\otimes n} \simeq H^{0}\left(K_{A^{n}}\right)$ for $A=V^{\vee} / \Lambda$, we have $\left(f_{n}\right)_{*} K_{f_{n}} \simeq L^{\otimes n}$ and

$$
K_{f_{n}} \simeq f_{n}^{*} L^{\otimes n} \text {. }
$$

2.2. Quotient by $\Gamma$. Let $\Gamma$ be a finite-index subgroup of the symplectic group $\operatorname{Sp}(\Lambda)$ of $\Lambda$. The quotient space $A(\Gamma)=\mathcal{D} / \Gamma$ is the Siegel modular variety defined by $\Gamma$. By Baily-Borel [2], $A(\Gamma)$ has the structure of a normal quasi-projective variety of dimension $g(g+1) / 2$.

The group $\Gamma$ acts on the vector bundle $\Lambda_{\mathbb{C}}$ equivariantly. This preserves $E$ and $\underline{\Lambda}$, and thus $\Gamma$ acts on $\mathcal{X}^{(n)}$. The quotient space

$$
X^{n}(\Gamma)=\mathcal{X}^{(n)} / \Gamma \simeq F^{\oplus n} /\left(\Lambda^{\oplus n} \rtimes \Gamma\right)
$$

is called the $n$-fold Kuga family. This is a normal quasi-projective variety of dimension $g(g+2 n+1) / 2$ fibered over $A(\Gamma)$. Here the quasi-projectivity follows from Mumford's GIT construction for the case $\Gamma=\operatorname{Sp}(\Lambda)$ ([18] Chapter 7, §2 - §3) and Grothendieck's Riemann existence theorem ([7] p. 442). For $n=1$ and some torsion-free $\Gamma$, Shimura [27] constructed a projective embedding of $X^{1}(\Gamma)$ using theta functions. In a special case in $g=2$, its defining equation is determined in [6]. General members of the fibration $X^{n}(\Gamma) \rightarrow A(\Gamma)$ are abelian varieties when $-1 \notin \Gamma$, and Kummer varieties when $-1 \in \Gamma$. We do not exclude the Kummer case.

The group $\Gamma$ acts on the line bundle $L$ equivariantly. A $\Gamma$-invariant section of $L^{\otimes k}$ is called a Siegel modular form of weight $k$ with respect to $\Gamma$. We write $M_{k}(\Gamma)$ for the space of them. We do not need to impose cusp condition by the Koecher principle (see, e.g., [5]).

2.3. Petersson metric. We fix an isomorphism $\operatorname{det} \Lambda \simeq \mathbb{Z}$. Let $[V] \in \mathcal{D}$. For two vectors $\omega=v_{1} \wedge \cdots \wedge v_{g}, \eta=w_{1} \wedge \cdots \wedge w_{g}$ of $L_{[V]}=\operatorname{det} V$, the wedge product

$$
\omega \wedge \bar{\eta}=v_{1} \wedge \cdots \wedge v_{g} \wedge \bar{w}_{1} \wedge \cdots \wedge \bar{w}_{g}
$$

is a vector of $\operatorname{det} \Lambda_{\mathbb{C}}$. We define the inner product of $\omega$ and $\eta$ to be the image of $i^{g^{2}} \omega \wedge \bar{\eta}$ in det $\Lambda_{\mathbb{C}} \simeq \mathbb{C}$. This defines a Hermitian metric on the line bundle $L$, which is $\operatorname{Sp}\left(\Lambda_{\mathbb{R}}\right)$-invariant by construction. Its $k$-th power defines an $\operatorname{Sp}\left(\Lambda_{\mathbb{R}}\right)$-invariant Hermitian metric on $L^{\otimes k}$ which we denote by $(,)_{k}$.

Geometrically, $(,)_{k}$ is the Hodge metric for $\mathcal{X}^{(k)} \rightarrow \mathcal{D}$.

Lemma 2.1. Let $A=V^{\vee} / \Lambda$ be the abelian variety over $[V] \in \mathcal{D}$. We identify $\omega, \eta \in L_{[V]}^{\otimes k}$ with canonical forms on $A^{k}$ by the natural isomorphism $L_{[V]}^{\otimes k} \simeq H^{0}\left(K_{A^{k}}\right)$. Then we have

$$
(\omega, \eta)_{k}=i^{g^{2} k} \int_{A^{k}} \omega \wedge \bar{\eta} .
$$


Proof. When $k=1$, the wedge product (2.1) corresponds to the $(g, g)$ form $\omega \wedge \bar{\eta}$ on $A$ via the isomorphism $\operatorname{det} \Lambda_{\mathbb{C}} \simeq H^{2 g}(A, \mathbb{C})$. The isomorphism $\operatorname{det} \Lambda_{\mathbb{C}} \simeq \mathbb{C}$ coincides with the integration map $\int_{A}: H^{2 g}(A, \mathbb{C}) \rightarrow \mathbb{C}$. When $k>1$, writing $\omega, \eta$ as $\omega=\omega_{0}^{\otimes k}$ and $\eta=\eta_{0}^{\otimes k}$ with $\omega_{0}, \eta_{0} \in L_{[V]}=H^{0}\left(K_{A}\right)$, we are reduced to the case $k=1$ by iterated integral.

We next define an invariant volume form. Via the isomorphism $K_{\mathcal{D}} \simeq$ $L^{\otimes g+1}$, the metric $(,)_{g+1}$ induces a Hermitian metric on $K_{\mathcal{D}}$. For each $[V] \in$ $\mathcal{D}$, we choose a vector $\omega \neq 0 \in\left(K_{\mathcal{D}}\right)_{[V]}$ and define

$$
\left(\operatorname{vol}_{\mathcal{D}}\right)_{[V]}=i^{N^{2}} \frac{\omega \wedge \bar{\omega}}{(\omega, \omega)_{g+1}}
$$

where $N=g(g+1) / 2$. This does not depend on the choice of $\omega$ and defines a volume form on $\mathcal{D}$, which is $\operatorname{Sp}\left(\Lambda_{\mathbb{R}}\right)$-invariant by the invariance of $(,)_{g+1}$. If $\omega, \eta$ are two local sections of $K_{\mathcal{D}}$ over some subset of $\mathcal{D}$, then

$$
(\omega, \eta)_{g+1} \operatorname{vol}_{\mathcal{D}}=i^{N^{2}} \omega \wedge \bar{\eta}
$$

2.4. Siegel upper half space. The traditional style defining Siegel modular forms as functions on the Siegel upper half space can be realized if we pick up a 0 -dimensional cusp of $\mathcal{D}$. Let $J$ be a maximal isotropic sublattice of $\Lambda$. We choose a maximal isotropic subspace $J_{\mathbb{Q}}^{\prime}$ of $\Lambda_{\mathbb{Q}}$ such that $\Lambda_{\mathbb{Q}}=J_{\mathbb{Q}} \oplus J_{\mathbb{Q}}^{\prime}$. (The role of $J_{\mathbb{Q}}^{\prime}$ will be auxiliary.) We can identify $J_{\mathbb{Q}}^{\prime} \simeq J_{\mathbb{Q}}^{\vee}$ and $\left(J_{\mathbb{Q}}^{\prime}\right)^{\vee} \simeq J_{\mathbb{Q}}$ by the symplectic pairing. $J$ determines the Zariski open set

$$
H_{J}=\left\{[V] \in \operatorname{LG}\left(\Lambda_{\mathbb{C}}\right) \mid V \cap J_{\mathbb{C}}=\{0\}\right\}
$$

of $\operatorname{LG}\left(\Lambda_{\mathbb{C}}\right)$. The choice of $J_{\mathbb{Q}}^{\prime}$ then induces an isomorphism $H_{J} \simeq \operatorname{Sym}^{2} J_{\mathbb{C}}$ by associating to an element of $\operatorname{Sym}^{2} J_{\mathbb{C}} \subset J_{\mathbb{C}} \otimes J_{\mathbb{C}} \simeq \operatorname{Hom}\left(J_{\mathbb{C}}^{\prime}, J_{\mathbb{C}}\right)$ its graph in $J_{\mathbb{C}}^{\prime} \oplus J_{\mathbb{C}}=\Lambda_{\mathbb{C}}$. (Symmetricity corresponds to isotropicity of the graph.) The domain $\mathcal{D}$ is contained in $H_{J}$, and its image by $H_{J} \rightarrow \mathrm{Sym}^{2} J_{\mathbb{C}}$ is

$$
\mathfrak{H}_{J}=\left\{\Omega \in \operatorname{Sym}^{2} J_{\mathbb{C}} \mid \operatorname{Im} \Omega>0\right\} .
$$

This is realization of $\mathcal{D}$ as a Siegel upper half space. If we change $J_{\mathbb{Q}}^{\prime}$, the isomorphism $H_{J} \rightarrow \mathrm{Sym}^{2} J_{\mathbb{C}}$ is shifted by translation.

We choose an orientation of $J$. This determines a generator of $\bigwedge^{g} J \simeq \mathbb{Z}$ which we denote by det $J$. Then we can define a nowhere vanishing section $s_{J}$ of the line bundle $L$ by the condition

$$
\left(s_{J}([V]), \operatorname{det} J\right)=1, \quad[V] \in \mathcal{D} .
$$

Here $\left(\right.$, ) is the paring between $L_{[V]}=\operatorname{det} V \subset \Lambda^{g} \Lambda_{\mathbb{C}}$ and $\operatorname{det} J \in \Lambda^{g} \Lambda_{\mathbb{C}}$ induced from the symplectic form on $\Lambda_{\mathbb{C}}$. The factor of automorphy associated to the frame $s_{J}$ is given by

$$
j(\gamma,[V])=\frac{\gamma\left(s_{J}([V])\right)}{s_{J}([\gamma V])}=\left(\gamma\left(s_{J}([V])\right), \operatorname{det} J\right) .
$$


Via the trivialization of $L^{\otimes k}$ by $s_{J}^{\otimes k}$, Siegel modular forms of weight $k$ are identified with holomorphic functions $F$ on $\mathcal{D}$ satisfying

$$
F([\gamma V])=j(\gamma,[V])^{k} F([V]), \quad \gamma \in \Gamma, \quad[V] \in \mathcal{D} .
$$

Over $\mathfrak{H}_{J}, j(\gamma,[V])$ takes the classical form as follows. Choose a basis $l_{1}, \cdots, l_{g}$ of $J$ of positive orientation, and let $m_{1}, \cdots, m_{g} \in J_{\mathbb{Q}}^{\prime}$ be its dual basis, namely $\left(m_{i}, l_{j}\right)=\delta_{i j}$. For $[V] \in \mathcal{D}$ we can take the basis $\omega_{1}, \cdots, \omega_{g}$ of $V$ such that $\left(\omega_{i}, l_{j}\right)=\delta_{i j}$ (normalized basis). If we write the matrix expression of $\left(\omega_{1}, \cdots, \omega_{g}\right)$ with respect to $\left(l_{i}\right)_{i},\left(m_{j}\right)_{j}$ in the form ${ }^{t}\left(\Omega I_{g}\right)$, then $\Omega$ is the symmetric matrix representing the image of $[V]$ in $\operatorname{Sym}^{2} J_{\mathbb{C}}$ with respect to $\left(l_{i}\right)_{i}$. Let $\left(\begin{array}{ll}A & B \\ C & D\end{array}\right)$ be the matrix representation of $\gamma$ with respect to $\left(l_{i}\right)_{i},\left(m_{j}\right)_{j}$. Since $s_{J}([V])=\omega_{1} \wedge \cdots \wedge \omega_{g}$, we have

$$
j(\gamma,[V])=\left(\gamma \omega_{1} \wedge \cdots \wedge \gamma \omega_{g}, l_{1} \wedge \cdots \wedge l_{g}\right)=\operatorname{det}(C \Omega+D) .
$$

We also calculate the Petersson metric on $L$ over $\mathfrak{H}_{J}$.

Lemma 2.2. Let $\Omega$ be the matrix expression of the image of $[V] \in \mathcal{D}$ in $\mathfrak{H}_{J}$. Then we have

$$
\left(s_{J}([V]), s_{J}([V])\right)_{1}=\operatorname{det}(\operatorname{Im} \Omega)
$$

up to a constant independent of $V$.

Proof. We use the notation above. Since $s_{J}([V])=\omega_{1} \wedge \cdots \wedge \omega_{g}$ and

$$
\omega_{1} \wedge \cdots \wedge \omega_{g} \wedge \bar{\omega}_{1} \wedge \cdots \wedge \bar{\omega}_{g}=\operatorname{det}\left(\begin{array}{cc}
\Omega & \bar{\Omega} \\
I_{g} & I_{g}
\end{array}\right) l_{1} \wedge \cdots \wedge l_{g} \wedge m_{1} \wedge \cdots \wedge m_{g},
$$

then $\left(s_{J}([V]), s_{J}([V])\right)_{1}$ equals to a constant multiple of

$$
\operatorname{det}\left(\begin{array}{ll}
\Omega & \bar{\Omega} \\
I_{g} & I_{g}
\end{array}\right)=\operatorname{det}\left(\begin{array}{cc}
\Omega-\bar{\Omega} & \bar{\Omega} \\
O & I_{g}
\end{array}\right)=(2 i)^{g} \operatorname{det}(\operatorname{Im} \Omega)
$$

Finally, we express $\operatorname{vol}_{\mathcal{D}}$ in terms of the flat volume form on $\mathrm{Sym}^{2} J_{\mathbb{C}}$. This recovers the classical form of Petersson inner product.

Lemma 2.3. Let $\mathrm{vol}_{J}$ be a flat volume form on $\mathrm{Sym}^{2} J_{\mathbb{C}}$. Under the isomorphism $\mathcal{D} \simeq \mathfrak{H}_{J}$ we have up to a constant

$$
\operatorname{vol}_{\mathcal{D}}=\frac{1}{\operatorname{det}(\operatorname{Im} \Omega)^{g+1}} \operatorname{vol}_{J}
$$

Proof. The canonical form $\omega_{J}$ on $\mathcal{D}$ corresponding to the section $s_{J}^{\otimes g+1}$ of $L^{\otimes g+1}$ extends to a translation-invariant canonical form on $\mathrm{Sym}^{2} J_{\mathbb{C}}$. Hence $\omega_{J}=d z_{1} \wedge \cdots \wedge d z_{N}$ for some coordinate $z_{1}, \cdots, z_{N}$ on $\operatorname{Sym}^{2} J_{\mathbb{C}}$. Then

$$
\operatorname{vol}_{\mathcal{D}}=i^{N^{2}} \frac{\omega_{J} \wedge \bar{\omega}_{J}}{\left(\omega_{J}, \omega_{J}\right)_{g+1}}=\frac{\operatorname{vol}_{J}}{\left(s_{J}, s_{J}\right)_{g+1}}=\frac{\operatorname{vol}_{J}}{\operatorname{det}(\operatorname{Im} \Omega)^{g+1}} .
$$




\section{Siegel domain REalization}

In this section we recall the Siegel domain realization (of the third kind) of $\mathcal{D}$ associated to each cusp. We give a self-contained description, following the style of [15], that is more explicit than the general theory, and that does not depend on coordinates so as to be suitable for dealing with general $\Gamma$ other than $\operatorname{Sp}(2 g, \mathbb{Z})$.

We fix a primitive isotropic sublattice $I$ of $\Lambda$, say of rank $g^{\prime}$, which corresponds to a cusp of $\mathcal{D}$. We set $g^{\prime \prime}=g-g^{\prime}$. We write $\Lambda(I)=I^{\perp} / I$, which is a nondegenerate symplectic lattice of rank $2 g^{\prime \prime}$.

3.1. Structure of the stabilizer. Let $\Gamma(I)_{\mathbb{Q}}$ be the stabilizer of $I_{\mathbb{Q}}$ in $\operatorname{Sp}\left(\Lambda_{\mathbb{Q}}\right)$. We define $U(I)_{\mathbb{Q}} \triangleleft W(I)_{\mathbb{Q}} \triangleleft \Gamma(I)_{\mathbb{Q}}$ by

$$
\begin{gathered}
U(I)_{\mathbb{Q}}=\operatorname{Ker}\left(\Gamma(I)_{\mathbb{Q}} \rightarrow \mathrm{GL}\left(I_{\mathbb{Q}}^{\perp}\right)\right), \\
W(I)_{\mathbb{Q}}=\operatorname{Ker}\left(\Gamma(I)_{\mathbb{Q}} \rightarrow \operatorname{Sp}\left(\Lambda(I)_{\mathbb{Q}}\right) \times \operatorname{GL}\left(I_{\mathbb{Q}}\right)\right),
\end{gathered}
$$

and put $V(I)_{\mathbb{Q}}=W(I)_{\mathbb{Q}} / U(I)_{\mathbb{Q}}$. The canonical exact sequence

$$
1 \rightarrow W(I)_{\mathbb{Q}} \rightarrow \Gamma(I)_{\mathbb{Q}} \rightarrow \operatorname{Sp}\left(\Lambda(I)_{\mathbb{Q}}\right) \times \mathrm{GL}\left(I_{\mathbb{Q}}\right) \rightarrow 1
$$

splits (non-canonically) if we choose an isotropic subspace $I_{\mathbb{Q}}^{\prime}$ of $\Lambda_{\mathbb{Q}}$ with $\Lambda_{\mathbb{Q}}=I_{\mathbb{Q}}^{\perp} \oplus I_{\mathbb{Q}}^{\prime}$ and let $\operatorname{Sp}\left(\Lambda(I)_{\mathbb{Q}}\right)$ act on $\left(I_{\mathbb{Q}} \oplus I_{\mathbb{Q}}^{\prime}\right)^{\perp} \simeq \Lambda(I)_{\mathbb{Q}}$ and $\operatorname{GL}\left(I_{\mathbb{Q}}\right)$ act on $I_{\mathbb{Q}} \oplus I_{\mathbb{Q}}^{\prime} \simeq I_{\mathbb{Q}} \oplus I_{\mathbb{Q}}^{\vee}$.

Elements of $W(I)_{\mathbb{Q}}$ can be described as follows. For $m \in I_{\mathbb{Q}}^{\perp}$ and $l \in I_{\mathbb{Q}}$ we define $T_{m, l} \in \operatorname{Sp}\left(\Lambda_{\mathbb{Q}}\right)$ by

$$
T_{m, l}(v)=v+(m, v) l+(l, v) m, \quad v \in \Lambda_{\mathbb{Q}} .
$$

Then $T_{m, l} \in W(I)_{\mathbb{Q}}$. The following relations hold:

(1) $T_{\alpha m, l}=T_{m, \alpha l}$ for $\alpha \in \mathbb{Q}$.

(2) $T_{m, l} \circ T_{m, l^{\prime}}=T_{m, l+l^{\prime}}$.

(3) $T_{m, l} \circ T_{m^{\prime}, l}=T_{l, \alpha l} \circ T_{m+m^{\prime}, l}$ where $\alpha=\left(m, m^{\prime}\right) / 2$.

(4) $T_{l, l^{\prime}}=T_{l^{\prime}, l}$ if $l, l^{\prime} \in I_{\mathbb{Q}}$.

Lemma 3.1. (1) The group $W(I)_{\mathbb{Q}}$ is generated by the elements $T_{m, l}$.

(2) We have the canonical isomorphisms

$$
\begin{array}{rlrl}
\operatorname{Sym}^{2} I_{\mathbb{Q}} & \simeq U(I)_{\mathbb{Q}}, & l \cdot l^{\prime} \mapsto T_{l, l^{\prime}}, \\
\Lambda(I)_{\mathbb{Q}} \otimes I_{\mathbb{Q}} & \simeq V(I)_{\mathbb{Q}}, & m \otimes l & \mapsto\left[T_{\tilde{m}, l}\right],
\end{array}
$$

where $\tilde{m} \in I_{\mathbb{Q}}^{\perp}$ is a lift of $m \in \Lambda(I)_{\mathbb{Q}}$. In particular, $U(I)_{\mathbb{Q}}$ and $V(I)_{\mathbb{Q}}$ are $\mathbb{Q}$-vector spaces.

Proof. This can be checked by choosing an isotropic subspace $I_{\mathbb{Q}}^{\prime} \subset \Lambda_{\mathbb{Q}}$ with $\Lambda_{\mathbb{Q}}=I_{\mathbb{Q}}^{\perp} \oplus I_{\mathbb{Q}}^{\prime}$ and calculating the action on $I_{\mathbb{Q}}^{\prime}$ and $\left(I_{\mathbb{Q}} \oplus I_{\mathbb{Q}}^{\prime}\right)^{\perp}$. 
Thus $W(I)_{\mathbb{Q}}$ is the unipotent radical of $\Gamma(I)_{\mathbb{Q}}, U(I)_{\mathbb{Q}}$ is the center of $W(I)_{\mathbb{Q}}$, and we have the exact sequence

$$
0 \rightarrow \operatorname{Sym}^{2} I_{\mathbb{Q}} \rightarrow W(I)_{\mathbb{Q}} \rightarrow \Lambda(I)_{\mathbb{Q}} \otimes I_{\mathbb{Q}} \rightarrow 0 .
$$

Since $U(I)_{\mathbb{Q}}$ is a normal subgroup of $\Gamma(I)_{\mathbb{Q}}$, we have the adjoint action of $\Gamma(I)_{\mathbb{Q}}$ on $U(I)_{\mathbb{Q}}$. Since $\gamma \circ T_{m, l} \circ \gamma^{-1}=T_{\gamma m, \gamma l}$ for $\gamma \in \Gamma(I)_{\mathbb{Q}}$, this coincides with the natural action of $\Gamma(I)_{\mathbb{Q}}$ on $\operatorname{Sym}^{2} I_{\mathbb{Q}}$.

Now let $\Gamma$ be a finite-index subgroup of $\operatorname{Sp}(\Lambda)$ and $\Gamma(I)_{\mathbb{Z}}=\Gamma(I)_{\mathbb{Q}} \cap \Gamma$ be the stabilizer of $I$ in $\Gamma$. We put

$$
W(I)_{\mathbb{Z}}=W(I)_{\mathbb{Q}} \cap \Gamma, \quad U(I)_{\mathbb{Z}}=U(I)_{\mathbb{Q}} \cap \Gamma, \quad V(I)_{\mathbb{Z}}=W(I)_{\mathbb{Z}} / U(I)_{\mathbb{Z}} .
$$

Then $U(I)_{\mathbb{Z}}$ is a lattice in $U(I)_{\mathbb{Q}} \simeq \operatorname{Sym}^{2} I_{\mathbb{Q}}$, and $V(I)_{\mathbb{Z}}$ is a lattice in $V(I)_{\mathbb{Q}} \simeq$ $\Lambda(I)_{\mathbb{Q}} \otimes I_{\mathbb{Q}}$. We also set

$$
{\overline{\Gamma(I)_{\mathbb{Z}}}}=\Gamma(I)_{\mathbb{Z}} / U(I)_{\mathbb{Z}}, \quad \Gamma_{I}=\Gamma(I)_{\mathbb{Z}} / W(I)_{\mathbb{Z}} .
$$

Then $\Gamma_{I}$ is mapped injectively into $\operatorname{Sp}(\Lambda(I)) \times \mathrm{GL}(I)$. By definition we have the canonical exact sequences

$$
\begin{gathered}
0 \rightarrow U(I)_{\mathbb{Z}} \rightarrow W(I)_{\mathbb{Z}} \rightarrow V(I)_{\mathbb{Z}} \rightarrow 0, \\
0 \rightarrow W(I)_{\mathbb{Z}} \rightarrow \Gamma(I)_{\mathbb{Z}} \rightarrow \Gamma_{I} \rightarrow 0 .
\end{gathered}
$$

3.2. Siegel domain realization. The choice of $I_{\mathbb{C}}$ determines the 2-step projection $\operatorname{LG}\left(\Lambda_{\mathbb{C}}\right) \rightarrow \operatorname{LG}\left(g^{\prime \prime}, I_{\mathbb{C}}^{\perp}\right) \rightarrow \mathrm{LG}\left(\Lambda(I)_{\mathbb{C}}\right)$. We shall show that restriction of this to $\mathcal{D} \subset \operatorname{LG}\left(\Lambda_{\mathbb{C}}\right)$ defines an embedded 2-step fibration

$$
\mathcal{D} \hookrightarrow \mathcal{D}(I) \hookrightarrow \operatorname{LG}\left(\mathcal{K}_{I}\right) \stackrel{\pi_{1}}{\rightarrow} \mathcal{V}_{I} \stackrel{\pi_{2}}{\rightarrow} \mathcal{D}_{\Lambda(I)}
$$

where

- $\mathcal{D}_{\Lambda(I)}$ is the Hermitian symmetric domain attached to $\Lambda(I)$,

- $\mathcal{V}_{I} \rightarrow \mathcal{D}_{\Lambda(I)}$ an affine space bundle for a vector bundle,

- $\mathrm{LG}\left(\mathcal{K}_{I}\right) \rightarrow \mathcal{V}_{I}$ a relative Lagrangian Grassmannian,

- $\mathcal{D}(I) \rightarrow \mathcal{V}_{I}$ a principal $\mathrm{Sym}^{2} I_{\mathbb{C}}$-bundle, and

- $\mathcal{D} \rightarrow \mathcal{V}_{I}$ a Siegel upper half space bundle.

This is an explicit form of the Siegel domain realization of $\mathcal{D}$ at $I$.

We define $\mathcal{D}(I)$ and $\mathcal{V}_{I}$ by

$$
\begin{aligned}
& \mathcal{D}(I)=\left\{[V] \in \operatorname{LG}\left(\Lambda_{\mathbb{C}}\right)|i(\cdot, \cdot)|_{V \cap I_{\mathbb{C}}^{\perp}}>0\right\}, \\
& \mathcal{V}_{I}=\left\{[W] \in \operatorname{LG}\left(g^{\prime \prime}, I_{\mathbb{C}}^{\perp}\right)|i(\cdot, \cdot)|_{W}>0\right\} .
\end{aligned}
$$

We consider the linear algebra construction

$$
V \mapsto(W, V / W) \mapsto W \mapsto \operatorname{Im}\left(W \rightarrow \Lambda(I)_{\mathbb{C}}\right)
$$

for $[V] \in \mathcal{D}(I)$ where $W=V \cap I_{\mathbb{C}}^{\perp}$. Then $[W] \in \mathcal{V}_{I}$. 
Lemma 3.2. Let $\Lambda(W)=\left(W^{\perp} \cap \Lambda_{\mathbb{C}}\right) / W$ and $I_{W} \subset \Lambda(W)$ be the image of $I_{\mathbb{C}}$, which is a maximal isotropic subspace of $\Lambda(W)$. Then we have $(V / W) \cap$ $I_{W}=\{0\}$ in $\Lambda(W)$. Conversely, if $\tilde{V} \cap I_{W}=\{0\}$ for $[\tilde{V}] \in \operatorname{LG}(\Lambda(W))$, then $\tilde{V}=V^{\prime} / W$ for some $\left[V^{\prime}\right] \in \mathcal{D}(I)$ containing $W$.

Proof. This is straightforward linear algebra.

Let $\mathcal{K}_{I} \rightarrow \mathcal{V}_{I}$ be the symplectic vector bundle whose fiber over $[W] \in \mathcal{V}_{I}$ is $\Lambda(W)=W^{\perp} / W$, and $\operatorname{LG}\left(\mathcal{K}_{I}\right)=\cup_{[W]} \operatorname{LG}(\Lambda(W))$ be its relative Lagrangian Grassmannian. The construction (3.3) defines the fibration

$$
\mathcal{D}(I) \hookrightarrow \operatorname{LG}\left(\mathcal{K}_{I}\right) \stackrel{\pi_{1}}{\rightarrow} \mathcal{V}_{I} \stackrel{\pi_{2}}{\rightarrow} \mathcal{D}_{\Lambda(I)}
$$

Proposition 3.3. (1) The image of $\mathcal{D}(I) \hookrightarrow \mathrm{LG}\left(\mathcal{K}_{I}\right)$ is a principal $U(I)_{\mathbb{C}^{-}}$ bundle over $\mathcal{V}_{I}$. For each $[W] \in \mathcal{V}_{I}, \mathcal{D} \cap \pi_{1}^{-1}([W])$ is a translation of the Siegel upper half space $\mathfrak{H}_{I}$ in $U(I)_{\mathbb{C}} \simeq \operatorname{Sym}^{2} I_{\mathbb{C}}$.

(2) $\mathcal{V}_{I} \rightarrow \mathcal{D}_{\Lambda(I)}$ is an affine space bundle for the vector bundle $F_{I} \otimes I_{\mathbb{C}}$ where $F_{I}$ is the universal quotient bundle over $\mathcal{D}_{\Lambda(I)}$. A choice of a lift $\Lambda(I)_{\mathbb{C}} \hookrightarrow I_{\mathbb{C}}^{\perp}$ of $\Lambda(I)_{\mathbb{C}}$ determines a section of $\mathcal{V}_{I} \rightarrow \mathcal{D}_{\Lambda(I)}$.

Proof. (1) By Lemma 3.2, $\mathcal{D}(I) \cap \pi_{1}^{-1}([W])$ coincides with the Zariski open set $\left\{[\tilde{V}] \mid \tilde{V} \cap I_{W}=\{0\}\right\} \simeq \operatorname{Sym}^{2} I_{W}$ of $\operatorname{LG}(\Lambda(W))$, on which $U(I)_{\mathbb{C}} \simeq \operatorname{Sym}^{2} I_{\mathbb{C}}$ acts by translation. The second assertion is similar to the case $g^{\prime}=g$ in $\$ 2.4$.

(2) Let $[U] \in \mathcal{D}_{\Lambda(I)}$ and $U^{\prime} \subset I_{\mathbb{C}}^{\perp}$ be its inverse image. Then

$$
\pi_{2}^{-1}([U])=\left\{W \subset U^{\prime} \mid \operatorname{dim} W=g^{\prime \prime}, W \cap I_{\mathbb{C}}=\{0\}\right\} .
$$

If we choose a lift $U^{\prime} \simeq I_{\mathbb{C}} \oplus U$ of $U$, we obtain an isomorphism $\pi_{2}^{-1}([U]) \simeq$ $\operatorname{Hom}\left(U, I_{\mathbb{C}}\right)$ by taking the graph of linear maps $U \rightarrow I_{\mathbb{C}}$. A lift of $\Lambda(I)_{\mathbb{C}}$ determines a lift of every $U$ and hence a section of $\pi_{2}$.

We have thus obtained a 2-step fibration as in (3.2). Finally, we describe the action of $\Gamma(I)_{\mathbb{Z}}$. We consider in three steps: first by $U(I)_{\mathbb{Z}}$, then by $V(I)_{\mathbb{Z}}$, and finally by $\Gamma_{I}$. Let $T_{I}=U(I)_{\mathbb{C}} / U(I)_{\mathbb{Z}}$ be the algebraic torus associated with the lattice $U(I)_{\mathbb{Z}}$. We have $\mathfrak{H}_{I} / U(I)_{\mathbb{Z}}=\operatorname{ord}^{-1}\left(C_{I}\right)$ inside $T_{I}$, where $C_{I} \subset \operatorname{Sym}^{2} I_{\mathbb{R}}$ is the cone of positive definite forms and ord: $T_{I} \rightarrow U(I)_{\mathbb{R}}$ is the projection map as in [1] p.2.

Proposition 3.4. (1) The quotient $\mathcal{T}_{I}=\mathcal{D}(I) / U(I)_{\mathbb{Z}}$ is a principal $T_{I}$-bundle over $\mathcal{V}_{I}$, and $\mathcal{B}_{I}=\mathcal{D} / U(I)_{\mathbb{Z}}$ is a $\operatorname{ord}^{-1}\left(C_{I}\right)$-bundle inside it.

(2) The group $V(I)_{\mathbb{Z}}$ acts on $\mathcal{D}_{\Lambda(I)}$ trivially, and on the fibers of $\mathcal{V}_{I} \simeq$ $F_{I} \otimes I_{\mathbb{C}}$ by translation by the lattice $V(I)_{\mathbb{Z}}$ of $\Lambda(I)_{\mathbb{Q}} \otimes I_{\mathbb{Q}}$. Thus $\mathcal{V}_{I} / V(I)_{\mathbb{Z}}$ is a fibration of abelian varieties over $\mathcal{D}_{\Lambda(I)}$.

(3) The group $\Gamma_{I}$ acts on $\mathcal{V}_{I} / V(I)_{\mathbb{Z}} \rightarrow \mathcal{D}_{\Lambda(I)}$ by the equivariant action of $\mathrm{Sp}(\Lambda(I)) \times \mathrm{GL}(I)$ on $F_{I} \otimes I_{\mathbb{C}}$ plus translation on the fibers. 
Proof. (1) follows from Proposition 3.3. (2) can be seen by expressing points of $\pi_{2}^{-1}([U])$ as graphs of $U \rightarrow I_{\mathbb{C}}$ and calculating the action of $T_{l, m}$ on them. (3) can be seen by choosing an isotropic subspace $I_{\mathbb{Q}}^{\prime}$ as before and the corresponding sections of (3.1) and of $\mathcal{V}_{I} \rightarrow \mathcal{D}_{\Lambda(I)}$. The translation is given by the difference of the two lifts of an element of $\Gamma_{I}$ in $\Gamma(I)_{\mathbb{Z}}$ and in the section of (3.1).

\section{TOROIDAL COMPACTIFICATION}

In this section we recall toroidal compactification of $A(\Gamma)$ following [1], [9]. We denote by $T(N)=N_{\mathbb{C}} / N$ the algebraic torus associated to a free $\mathbb{Z}$-module $N$ of finite rank. We especially write $T_{I}=T\left(U(I)_{\mathbb{Z}}\right)$.

4.1. Relative torus embedding. Let $I$ be a primitive isotropic sublattice of $\Lambda$. We equip $U(I)_{\mathbb{R}} \simeq \operatorname{Sym}^{2} I_{\mathbb{R}}$ with a $\mathbb{Z}$-structure by $U(I)_{\mathbb{Z}}$. Let $C_{I} \subset$ $U(I)_{\mathbb{R}}$ be the cone of positive definite forms on $I_{\mathbb{R}}^{\vee}$, and $C_{I}^{*} \subset U(I)_{\mathbb{R}}$ the cone of semi positive definite forms whose kernel is defined over $\mathbb{Q}$. In other words, $C_{I}^{*}=\bigcup_{I^{\prime}} C_{I^{\prime}}$ where $I^{\prime}$ ranges over all primitive sublattices of $I$ (including $I^{\prime}=\{0\}$ ). Recall that $\Gamma(I)_{\mathbb{R}}$ acts on $U(I)_{\mathbb{R}}$ by the adjoint action which coincides with the natural action on $\operatorname{Sym}^{2} I_{\mathbb{R}}$. A fan $\Sigma=\left(\sigma_{\alpha}\right)_{\alpha}$ in $U(I)_{\mathbb{R}}$ is called $\Gamma(I)_{\mathbb{Z}}$-admissible if

(1) the support of $\Sigma$ is $C_{I}^{*}$,

(2) $\Sigma$ is preserved by the action of $\Gamma(I)_{\mathbb{Z}}$, and

(3) $\Sigma / \Gamma(I)_{\mathbb{Z}}$ consists of only finitely many cones.

Let $T_{I} \hookrightarrow T_{I}^{\Sigma}$ be the torus embedding defined by the fan $\Sigma$. A ray $\mathbb{R}_{\geq 0} Q$ in $\Sigma$ corresponds to a $T_{I}$-orbit of codimension 1 in the boundary of $T_{I}^{\Sigma}$, say $\Delta_{Q}$. We always take $Q$ to be a primitive vector of $U(I)_{\mathbb{Z}}$. Let $T_{I}^{Q}$ be the torus embedding of $T_{I}$ defined by the ray $\mathbb{R}_{\geq 0} Q$. Then $T_{I} \subset T_{I}^{Q} \subset T_{I}^{\Sigma}$ and $\Delta_{Q}$ is the unique boundary divisor of $T_{I}^{Q}$. Let $T_{Q}=T(\mathbb{Z} Q) \simeq \mathbb{C}^{\times}$and $\bar{T}_{Q} \simeq \mathbb{C}$ be its the standard partial compactification. Then $T_{I}^{Q} \simeq\left(T_{I} \times \bar{T}_{Q}\right) / T_{Q}$ and $\Delta_{Q} \simeq T_{I} / T_{Q} \simeq T\left(U(I)_{\mathbb{Z}} / \mathbb{Z} Q\right)$. The embedding $T_{Q} \hookrightarrow T_{I}$ extends to $\bar{T}_{Q} \hookrightarrow T_{I}^{Q}$ which gives a normal space of $\Delta_{Q}$ at its base point.

A character $e^{\chi}=\exp (2 \pi i \chi(\cdot))$ of $T_{I}$, where $\chi \in U(I)_{\mathbb{Z}}^{\vee}$, extends holomorphically over $\Delta_{Q}$ if and only if $\chi(Q) \geq 0$, and it is identically 0 at $\Delta_{Q}$ if and only if $\chi(Q)>0$. By restriction, the character group of $\Delta_{Q}$ is identified with $Q^{\perp} \cap U(I)_{\mathbb{Z}}^{\vee}$. If we choose $\chi \in U(I)_{\mathbb{Z}}^{\vee}$ such that $\chi(Q)=1$ (this is possible because $Q$ is primitive in $\left.U(I)_{\mathbb{Z}}\right)$, then $\Delta_{Q}$ is defined by $e^{\chi}=0$ and $e^{\chi}$ gives a normal parameter around $\Delta_{Q}$.

Now let $\mathcal{T}_{I} \rightarrow \mathcal{V}_{I}$ be the principal $T_{I}$-bundle constructed in Proposition 3.4. We can form the relative torus embedding $\mathcal{T}_{I}^{\Sigma}=\left(\mathcal{T}_{I} \times T_{I}^{\Sigma}\right) / T_{I}$. Let $\mathcal{B}_{I}^{\Sigma}$ be the interior of the closure of $\mathcal{B}_{I}$ in $\mathcal{T}_{I}^{\Sigma}$. This is the partial compactification 
in the direction of $I$ defined by $\Sigma$. Since $\Gamma(I)_{\mathbb{Z}}$ preserves $\Sigma$, the $\overline{\Gamma(I)}_{\mathbb{Z}}$-action on $\mathcal{B}_{I}$ extends to the action on $\mathcal{B}_{I}^{\Sigma}$. This is properly discontinuous (cf. [1]).

4.2. Adjacent cusps. Let $J$ be a primitive isotropic sublattice of $\Lambda$ that contains $I$. The cusp of $\mathcal{D}$ associated to $J$ is in the closure of the cusp associated to $I$. We shall describe the relationship between the relative torus embeddings for $I$ and for $J$. First note that $U(I)_{\mathbb{R}} \simeq \operatorname{Sym}^{2} I_{\mathbb{R}}$ is contained in $U(J)_{\mathbb{R}} \simeq \operatorname{Sym}^{2} J_{\mathbb{R}}$. Then $U(I)_{\mathbb{Z}}$ is a primitive sublattice of $U(J)_{\mathbb{Z}}$, so $T_{I}$ is a sub torus of $T_{J}$. The cone $C_{I}^{*}$ is an extremal sub cone of $C_{J}^{*}$. If we have a fan $\Sigma_{J}$ in $U(J)_{\mathbb{R}}$ with support $C_{J}^{*}$, its restriction $\Sigma_{I}=\left.\Sigma_{J}\right|_{I}$ to $U(I)_{\mathbb{R}}$ is a fan in $U(I)_{\mathbb{R}}$ with support $C_{I}^{*}$. Here $\left.\Sigma_{J}\right|_{I}$ consists of cones $\sigma_{\alpha}$ in $\Sigma_{J}$ with $\sigma_{\alpha} \subset C_{I}^{*}$. The embedding $T_{I} \hookrightarrow T_{J}$ extends to $T_{I}^{\Sigma_{I}} \hookrightarrow T_{J}^{\Sigma_{J}}$.

We set ${\overline{U(J)_{\mathbb{Z}}}}=U(J)_{\mathbb{Z}} / U(I)_{\mathbb{Z}}$. We have the quotient map

$$
\mathcal{B}_{I}=\mathcal{D} / U(I)_{\mathbb{Z}} \rightarrow \mathcal{B}_{J}=\mathcal{D} / U(J)_{\mathbb{Z}}
$$

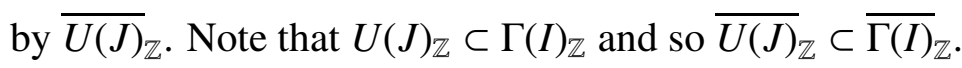

Lemma 4.1. For $I \subset J$ the quotient map $\mathcal{B}_{I} \rightarrow \mathcal{B}_{J}$ extends to an etale map $\mathcal{B}_{I}^{\Sigma_{I}} \rightarrow \mathcal{B}_{J}^{\Sigma_{J}}$. More specifically, it factorizes as

$$
\mathcal{B}_{I}^{\Sigma_{I}} \rightarrow \mathcal{B}_{I}^{\Sigma_{I}} / \overline{U(J)}_{\mathbb{Z}} \hookrightarrow \mathcal{B}_{J}^{\Sigma_{J}},
$$

where the first map is a free quotient map, and the second is an open immersion whose image does not intersect with the boundary strata of $\mathcal{B}_{J}^{\Sigma_{J}}$ corresponding to the cones in $\Sigma_{J}-\Sigma_{I}$.

Proof. Since $I \subset J \subset J^{\perp} \subset I^{\perp}$, we have $\mathcal{D}(I) \subset \mathcal{D}(J)$. This is clearly compatible with the action of $U(I)_{\mathbb{C}} \subset U(J)_{\mathbb{C}}$. Dividing by $U(I)_{\mathbb{Z}} \subset U(J)_{\mathbb{Z}}$, we obtain a map $\mathcal{T}_{I} \rightarrow \mathcal{T}_{J}$ which is compatible with the action of $T_{I} \hookrightarrow T_{J}$. Hence $\mathcal{T}_{I} \rightarrow \mathcal{T}_{J}$ extends to $\mathcal{T}_{I}^{\Sigma_{I}} \rightarrow \mathcal{T}_{J}^{\Sigma_{J}}$ whose restriction gives $\mathcal{B}_{I}^{\Sigma_{I}} \rightarrow \mathcal{B}_{J}^{\Sigma_{J}}$.

We shall observe $\mathcal{T}_{I}^{\Sigma_{I}} \rightarrow \mathcal{T}_{J}^{\Sigma_{J}}$ more closely. We put

$$
\mathcal{T}_{J}^{\Sigma_{I}}=\left(\mathcal{T}_{J} \times T_{J}^{\Sigma_{I}}\right) / T_{J} \simeq\left(\mathcal{T}_{J} \times T_{I}^{\Sigma_{I}}\right) / T_{I}
$$

Then $\mathcal{T}_{I}^{\Sigma_{I}} \rightarrow \mathcal{T}_{J}^{\Sigma_{J}}$ factorizes as

$$
\mathcal{T}_{I}^{\Sigma_{I}} \rightarrow \mathcal{T}_{J}^{\Sigma_{I}} \rightarrow \mathcal{T}_{J}^{\Sigma_{J}}
$$

The second map $\mathcal{T}_{J}^{\Sigma_{I}} \rightarrow \mathcal{T}_{J}^{\Sigma_{J}}$ is an open embedding, whose complement consists of boundary strata corresponding to the cones in $\Sigma_{J}-\Sigma_{I}$. As for the first map $\mathcal{T}_{I}^{\Sigma_{I}} \rightarrow \mathcal{T}_{J}^{\Sigma_{I}}$, note that $\mathcal{T}_{I} \rightarrow \mathcal{T}_{J}$ is a $T_{I}$-equivariant map between the principal $T_{I}$-bundles $\mathcal{T}_{I} \rightarrow \mathcal{V}_{I}$ and $\mathcal{T}_{J} \rightarrow \mathcal{T}_{J} / T_{I}$, The map between the bases $\mathcal{V}_{I} \rightarrow \mathcal{T}_{J} / T_{I}$ factorizes as

$\mathcal{V}_{I}=\mathcal{D}(I) / U(I)_{\mathbb{C}} \rightarrow \mathcal{D}(I) / U(I)_{\mathbb{C}}+U(J)_{\mathbb{Z}} \hookrightarrow \mathcal{D}(J) / U(I)_{\mathbb{C}}+U(J)_{\mathbb{Z}}=\mathcal{T}_{J} / T_{I}$. 
The first map is a free quotient by $\overline{U(J)}_{\mathbb{Z}}$, and the second is an open embedding. Thus $\mathcal{T}_{I}^{\Sigma_{I}} \rightarrow \mathcal{T}_{J}^{\Sigma_{I}}$ is also a composition of a free quotient by ${\overline{U(J)_{\mathbb{Z}}}}_{\mathbb{1}}$ and an open embedding.

4.3. Toroidal compactification. A toroidal compactification of $A(\Gamma)$ is constructed from the following data.

Definition 4.2 ([1],$[9])$. An admissible collection of fans for $\Gamma$ is a collection $\Sigma=\left(\Sigma_{I}\right)_{I}$ of fans, one for each primitive isotropic sublattice $I$ of $\Lambda$, which satisfies the following conditions:

(1) $\Sigma_{I}$ is a $\Gamma(I)_{\mathbb{Z}}$-admissible fan in $U(I)_{\mathbb{R}}$,

(2) $\gamma\left(\Sigma_{I}\right)=\Sigma_{\gamma I}$ for $\gamma \in \Gamma$, and

(3) when $I \subset J$, then $\left.\Sigma_{J}\right|_{I}=\Sigma_{I}$.

We will abbreviate $\mathcal{B}_{I}^{\Sigma_{I}}=\mathcal{B}_{I}^{\Sigma}$ when no confusion is likely to occur. The toroidal compactification of $A(\Gamma)$ by $\Sigma$ is defined as ([1] [ [9])

$$
A(\Gamma)^{\Sigma}=\left(\bigsqcup_{I} \mathcal{B}_{I}^{\Sigma_{I}}\right) / \sim,
$$

where $I$ ranges over all primitive isotropic sublattices of $\Lambda$ (including $I=$ $\{0\}$ where $\left.\mathcal{B}_{I}=\mathcal{B}_{I}^{\Sigma_{I}}=\mathcal{D}\right)$, and $\sim$ is the equivalence relation generated by the following relations:

(1) the isomorphism $\gamma: \mathcal{B}_{I}^{\Sigma_{I}} \rightarrow \mathcal{B}_{\gamma I}^{\Sigma_{\gamma I}}$ by $\gamma \in \Gamma$, and

(2) the etale map $\mathcal{B}_{I}^{\Sigma_{I}} \rightarrow \mathcal{B}_{J}^{\Sigma_{J}}$ for $I \subset J$ as in Lemma 4.1.

Let $\Sigma_{I}^{\circ}=\Sigma_{I} \backslash \cup_{K \subsetneq I} \Sigma_{K}$ be the set of cones in $\Sigma_{I}$ whose relative interior is contained in $C_{I}$. We write $\Delta_{\sigma, I}$ for the boundary stratum of $\mathcal{B}_{I}^{\Sigma}$ corresponding to a cone $\sigma \in \Sigma_{I}$, and let $\Delta_{I}=\bigcup_{\sigma \in \Sigma_{I}^{\circ}} \Delta_{\sigma, I}$ be the union of boundary strata that does not come from higher dimensional cusps adjacent to $I$. By Lemma 4.1, the natural map $\Delta_{I} / \overline{\Gamma(I)}_{\mathbb{Z}} \rightarrow A(\Gamma)^{\Sigma}$ is injective.

Theorem 4.3 ([1]]). Let $\Sigma$ be an admissible collection of fans for $\Gamma$.

(1) $A(\Gamma)^{\Sigma}$ is a compact Moishezon space containing $A(\Gamma)$ as a Zariski open set. We have a surjective morphism from $A(\Gamma)^{\Sigma}$ to the Satake compactification of $A(\Gamma)$, which maps $\Delta_{I}$ to the boundary component for I.

(2) For each primitive isotropic sublattice I of $\Lambda$, the natural map

$$
\mathcal{B}_{I}^{\Sigma} / \overline{\Gamma(I)}_{\mathbb{Z}} \rightarrow A(\Gamma)^{\Sigma}
$$

is isomorphic on an open neighborhood of $\Delta_{I} / \overline{\Gamma(I)}_{\mathbb{Z}}$.

By the property (2) (see [1] p. 175), the quotient space $\mathcal{B}_{I}^{\Sigma} / \overline{\Gamma(I)}_{\mathbb{Z}}$ gives a local model of $A(\Gamma)^{\Sigma}$ around the boundary strata lying over the $I$-cusp. 
4.4. Extension of the modular line bundle. There is a natural number $k^{\prime}$ such that for every $x \in \mathcal{D}$ and $\gamma \in \Gamma$ with $\gamma(x)=x, \gamma$ acts trivially on $L_{x}^{\otimes k^{\prime}}$. Then $L^{\otimes k^{\prime}}$ descends to a line bundle over $A(\Gamma)$. In this subsection we extend some multiple of this line bundle over $A(\Gamma)^{\Sigma}$. This is an explicit form of Mumford's extension [17]. We proceed in two steps: first extend $L$ from $\mathcal{B}_{I}$ to $\mathcal{B}_{I}^{\Sigma}$ for each $I$; then for some $k, L^{\otimes k}$ descends from $\sqcup_{I} \mathcal{B}_{I}^{\Sigma}$ to $A(\Gamma)^{\Sigma}$.

As the first step, let $I$ be a primitive isotropic sublattice of $\Lambda$. We choose a maximal isotropic sublattice $J \subset \Lambda$ containing $I$. Fix an orientation of $I$ and $J$. Let $s_{J}$ be the frame of $L$ over $\mathcal{D}$ associated to $J$ (see $\$ 2.4$ ). Since $s_{J}$ is invariant under $U(I)_{\mathbb{Z}} \subset U(J)_{\mathbb{Z}}$, it descends to a frame of $L$ over $\mathcal{B}_{I}=$ $\mathcal{D} / U(I)_{\mathbb{Z}}$ which we again denote by $s_{J}$. Then there exists a unique extension of $L$ to a line bundle over $\mathcal{B}_{I}^{\Sigma}$, denoted again by $L$, such that $s_{J}$ extends to its frame. A section $s$ of $L^{\otimes k}$ over $\mathcal{B}_{I}$ extends holomorphically over $\mathcal{B}_{I}^{\Sigma}$ if and only if the function $s / s_{J}^{\otimes k}$ over $\mathcal{B}_{I}$ extends holomorphically over $\mathcal{B}_{I}^{\Sigma}$.

Proposition 4.4. The extension of $L$ defined above is independent of the choice of $J$ up to isomorphism. Moreover, the equivariant action of $\overline{\Gamma(I)}_{\mathbb{Z}}$ on L over $\mathcal{B}_{I}$ extends to an equivariant action over $\mathcal{B}_{I}^{\Sigma}$.

In order to prove this, we consider a decomposition of $s_{J}$. Let

$$
\mathcal{D} \hookrightarrow \operatorname{LG}\left(\mathcal{K}_{I}\right) \stackrel{\pi_{1}}{\rightarrow} \mathcal{V}_{I} \stackrel{\pi_{2}}{\rightarrow} \mathcal{D}_{\Lambda(I)}
$$

be the Siegel domain realization with respect to $I$, and $\pi=\pi_{2} \circ \pi_{1}$. Let $E_{I} \rightarrow \mathcal{D}_{\Lambda(I)}$ be the universal sub bundle over $\mathcal{D}_{\Lambda(I)}$ and $L_{I}=\operatorname{det} E_{I}$. We have the frame $s_{J / I}$ of $L_{I}$ associated to the oriented, maximal isotropic sublattice $J / I$ of $\Lambda(I)$. On the other hand, we have the relative tautological bundle $E_{\pi_{1}}$ over $\operatorname{LG}\left(\mathcal{K}_{I}\right)$ whose fiber over $[V] \in \mathcal{D}$ is $V / W$ where $W=V \cap I_{\mathbb{C}}^{\perp}$. Let $L_{\pi_{1}}=\left.\operatorname{det} E_{\pi_{1}}\right|_{\mathcal{D}}$. We have the frame $s_{I}$ of $L_{\pi_{1}}$ defined by the condition $\left(s_{I}([V]), \operatorname{det} I\right)=1$, where $($,$) is the pairing between \operatorname{det}(V / W) \subset \Lambda^{g^{\prime}}(\Lambda(W))$ and $\operatorname{det} I_{W} \subset \wedge^{g^{\prime}}(\Lambda(W))$ induced from the symplectic form on $\Lambda(W)$.

Lemma 4.5. We have a natural isomorphism $L \simeq L_{\pi_{1}} \otimes \pi^{*} L_{I}$. Under this isomorphism we have $s_{J}=s_{I} \otimes \pi^{*} s_{J / I}$.

Proof. By varying the exact sequence of vector spaces

$$
0 \rightarrow V \cap I_{\mathbb{C}}^{\perp} \rightarrow V \rightarrow V /\left(V \cap I_{\mathbb{C}}^{\perp}\right) \rightarrow 0
$$

over $\mathcal{D}$, we obtain the exact sequence of vector bundles

$$
0 \rightarrow \pi^{*} E_{I} \rightarrow E \rightarrow E_{\pi_{1}} \rightarrow 0 .
$$

This shows that $L \simeq L_{\pi_{1}} \otimes \pi^{*} L_{I}$. Since

$$
\left(s_{I} \otimes \pi^{*} s_{J / I}, \operatorname{det} J\right)=\left(s_{I}, \operatorname{det} I\right) \cdot\left(s_{J / I}, \operatorname{det}(J / I)\right)=1,
$$

we have $s_{J}=s_{I} \otimes \pi^{*} s_{J / I}$. 
(Proof of Proposition 4.4). If $J^{\prime} \supset I$ is another maximal isotropic sublattice, then $s_{J} / s_{J^{\prime}}=\pi^{*}\left(s_{J / I} / s_{J^{\prime} / I}\right)$ is the pullback of a nowhere vanishing function on $\mathcal{D}_{\Lambda(I)}$. Since the partial compactification $\mathcal{B}_{I} \hookrightarrow \mathcal{B}_{I}^{\Sigma}$ is done relatively over $\mathcal{V}_{I}, s_{J} / s_{J^{\prime}}$ extends to a nowhere vanishing function on $\mathcal{B}_{I}^{\Sigma}$. This shows the independence of the extension from $J$. If we consider $J^{\prime}=\gamma J$ for $\gamma \in \Gamma(I)_{\mathbb{Z}}$, this also implies the second assertion.

We consider the collection of these extended line bundles over the whole $\sqcup_{I} \mathcal{B}_{I}^{\Sigma}$ and denote it again by $L$. The $\Gamma$-action on $L$ over $\sqcup_{I} \mathcal{B}_{I}$ extends over $\sqcup_{I} \mathcal{B}_{I}^{\Sigma}$ by Proposition 4.4. Furthermore, if $p: \mathcal{B}_{I}^{\Sigma} \rightarrow \mathcal{B}_{J}^{\Sigma}$ is the etale map for $I \subset J$ as in Lemma 4.1, the isomorphism $\left.p^{*}\left(\left.L\right|_{\mathcal{B}_{J}}\right) \simeq L\right|_{\mathcal{B}_{I}}$ over $\mathcal{B}_{I}$ extends over $\mathcal{B}_{I}^{\Sigma}$, because we can use a common frame $s_{K}$ for the extension over both $\mathcal{B}_{I}^{\Sigma}$ and $\mathcal{B}_{J}^{\Sigma}$ where $K$ is maximal with $K \supset J \supset I$.

Lemma 4.6 (cf. [17]). A modular form $F$ of weight $k$, as a section of $L^{\otimes k}$ over $\mathcal{B}_{I}$, extends holomorphically over $\mathcal{B}_{I}^{\Sigma} . F$ is a cusp form if and only if it vanishes at the boundary divisor of $\mathcal{B}_{I}^{\Sigma}$ for all $I$.

Proof. By the above gluing, we may assume that $I$ is maximal. We identify $F$ with a function on $\mathcal{B}_{I}$ via the frame $s_{I}^{\otimes k}$, which has Fourier expansion

$$
F=\sum_{\chi \in U(I)_{\mathbb{Z}}^{\vee}} a_{\chi} e^{\chi}, \quad e^{\chi}=\exp (2 \pi i \chi(\cdot)) .
$$

By the Koecher principle, we have $a_{\chi} \neq 0$ only when $\chi$ is semi positive definite. Then $\chi(Q) \geq 0$ for every ray $\mathbb{R}_{\geq 0} Q$ in $\Sigma_{I}$, so $e^{\chi}$ extends holomorphically over $\mathcal{B}_{I}^{\Sigma}$ for such $\chi$. This proves the first assertion.

By definition, $F$ is a cusp form if and only if $a_{\chi} \neq 0$ only for positive definite $\chi$ at all maximal $I$. Since $\chi$ is strictly semi positive definite if and only if $\chi \in Q^{\perp}$ for some ray $\mathbb{R}_{\geq 0} Q \in \Sigma_{I}$, the cuspidal condition is equivalent to $a_{\chi}=0$ for all $\chi \in Q^{\perp}$ for every ray $\mathbb{R}_{\geq 0} Q \in \Sigma_{I}$ at every maximal $I$. Since $Q^{\perp} \cap U(I)_{\mathbb{Z}}^{\vee}$ is the character group of the boundary torus associated to $\mathbb{R}_{\geq 0} Q$, this is equivalent to the vanishing of $F$ at the boundary of $\mathcal{B}_{I}^{\Sigma}$ for every maximal $I$.

We choose a natural number $k$ such that for every $I, x \in \mathcal{B}_{I}^{\Sigma}, \gamma \in \Gamma(I)_{\mathbb{Z}}$ with $\gamma(x)=x, \gamma$ acts trivially on $L_{x}^{\otimes k}$. Then the line bundle $L^{\otimes k}$ over $\sqcup_{I} \mathcal{B}_{I}^{\Sigma}$ descends to a line bundle over $A(\Gamma)^{\Sigma}=\left(\sqcup_{I} \mathcal{B}_{I}^{\Sigma}\right) / \sim$. This will be denoted as $L^{\otimes k}$ by abuse of notation (for $L$ might not exist as a line bundle over $A(\Gamma)^{\Sigma}$ ). By Lemma 4.6, we have $H^{0}\left(A(\Gamma)^{\Sigma}, L^{\otimes k}\right) \simeq M_{k}(\Gamma)$.

\section{Asymptotic estimate of Petersson norm}

Let $A(\Gamma)^{\Sigma}$ be a toroidal compactification of $A(\Gamma)$. Let $J$ be a maximal isotropic sublattice of $\Lambda$ and $\mathbb{R}_{\geq 0} Q$ be a ray in $\Sigma_{J}$. Let $\Delta_{Q}=\Delta_{Q, J}$ be the 
corresponding boundary stratum of $\mathcal{B}_{J}^{\Sigma}$. The image of $\Delta_{Q}$ in $A(\Gamma)^{\Sigma}$ is a Zariski open set of an irreducible component of the boundary divisor of $A(\Gamma)^{\Sigma}$. In this section we prepare an asymptotic estimate of the Petersson norm of a local modular form as the period approaches $\Delta_{Q}$.

We choose $\chi \in U(J)_{\mathbb{Z}}^{\vee}$ with $\chi(Q)=1$. Recall that $q=\exp (2 \pi i \chi(\cdot))$ gives a normal parameter around $\Delta_{Q}$. We take an arbitrary point $x$ of $\Delta_{Q}$ and a small neighborhood $\Delta_{x}$ of $x$ in $\Delta_{Q}$. Let $T_{r} \subset \mathcal{B}_{J}^{\Sigma}$ be the tubular neighborhood of $\Delta_{x}$ of radius $r$, defined by $|q| \leq r$. We fix a sufficiently small $0<R \ll 1$ and set $W_{\varepsilon}=T_{R}-T_{\varepsilon}$ for $0<\varepsilon<R$, which is the annulus bundle around $\Delta_{x}$ of radius $[\varepsilon, R]$. We want to give an asymptotic estimate of

$$
\int_{W_{\varepsilon}}(F, F)_{k}^{\beta} \operatorname{vol}_{\mathcal{D}} \quad(\varepsilon \rightarrow 0)
$$

for $F$ a local section of $L^{\otimes k}$ defined around $x$ and $\beta>0$. We first compute the asymptotic behavior of the Petersson metric on $L$.

Lemma 5.1. Let $s_{J}$ be the distinguished frame of L associated to J. Around each point $x$ of $\Delta_{Q}$, we have

$$
\left(s_{J}, s_{J}\right)_{1} \sim C_{x} \cdot(-\log |q|)^{\mathrm{rk}(Q)} \quad(|q| \rightarrow 0)
$$

for some constant $C_{x}>0$, where $\operatorname{rk}(Q)$ is the rank of $Q$ as a quadratic form.

Proof. We choose a maximal isotropic subspace $J_{\mathbb{Q}}^{\prime}$ of $\Lambda_{\mathbb{Q}}$ such that $\Lambda_{\mathbb{Q}}=$ $J_{\mathbb{Q}} \oplus J_{\mathbb{Q}}^{\prime}$. Recall that this induces an isomorphism $\iota: \mathcal{D} \rightarrow \mathfrak{H}_{J}$. We identify Sym $^{2} J_{\mathbb{C}}$ with the space of $n \times n$ symmetric matrices by taking a basis of $J_{\mathbb{Q}}$. By Lemma 2.2, if $\Omega=\iota([V])$ for $[V] \in \mathcal{D}$, then

$$
\left(s_{J}([V]), s_{J}([V])\right)_{1}=\operatorname{det}(\operatorname{Im} \Omega) .
$$

We pick up a point $\Omega_{0} \in \mathfrak{H}_{J}$ and consider the flow $\Omega_{t}=\Omega_{0}+$ it $Q$ in $\mathfrak{H}_{J}$, where $t \in \mathbb{R}_{>0}$. The image of $\Omega_{t}$ in $\mathcal{B}_{J}$ converges to a point of $\Delta_{Q}$, say $x$, from the normal direction. Then

$$
q=\exp \left(2 \pi i \chi\left(\Omega_{t}\right)\right)=\exp \left(2 \pi i \chi\left(\Omega_{0}\right)\right) \cdot \exp (-2 \pi t),
$$

so we have

$$
t \sim(-2 \pi)^{-1} \log |q| \quad(t \rightarrow+\infty) .
$$

This shows that

$$
\operatorname{det}\left(\operatorname{Im} \Omega_{t}\right) \sim C \cdot t^{\mathrm{rk}(Q)} \sim C \cdot(-\log |q|)^{\mathrm{rk}(Q)} \quad(t \rightarrow+\infty),
$$

where $C$ stands for any unspecified positive constant.

Note that if $Q$ belongs to $C_{I} \subset C_{J}^{+}$for $I \subset J$, then $\operatorname{rk}(Q)=\operatorname{rk}(I)$. Our main result of this section is the following. 
Proposition 5.2. Let $F$ be a local section of $L^{\otimes k}$ defined over a neighborhood of $x \in \Delta_{Q} \subset \mathcal{B}_{J}^{\Sigma}$. Let $\beta>0$ be a positive real number. Then

$$
\int_{W_{\varepsilon}}(F, F)_{k}^{\beta} \operatorname{vol}_{\mathcal{D}}=o\left(\varepsilon^{-\alpha}\right) \quad(\varepsilon \rightarrow 0)
$$

for every $\alpha>0$. Moreover, when $k \beta \geq g+1, F$ vanishes at $\Delta_{Q}$ if and only if

$$
\int_{W_{\varepsilon}}(F, F)_{k}^{\beta} \operatorname{vol}_{\mathcal{D}}=O(1) \quad(\varepsilon \rightarrow 0) .
$$

Proof. Via the frame $s_{J}^{\otimes k}$ we identify $F$ with a holomorphic function $F(\Omega)$ defined around $x$. Let $\operatorname{vol}_{J}$ be a flat volume form on $\mathrm{Sym}^{2} J_{\mathbb{C}}$. By Lemmas 2.2 and 2.3, we have

$$
\int_{W_{\varepsilon}}(F, F)_{k}^{\beta} \operatorname{vol}_{\mathcal{D}}=\int_{W_{\varepsilon}}|F(\Omega)|^{2 \beta} \cdot \operatorname{det}(\operatorname{Im} \Omega)^{k \beta-g-1} \operatorname{vol}_{J} .
$$

Locally around $x$, we can write (up to constant)

$$
\operatorname{vol}_{J}=d \chi \wedge d \bar{\chi} \wedge \operatorname{vol}_{\Delta_{x}}=|q|^{-2} d q \wedge d \bar{q} \wedge \operatorname{vol}_{\Delta_{x}}=r^{-1} d r \wedge d \theta \wedge \operatorname{vol}_{\Delta_{x}}
$$

for some volume form $\operatorname{vol}_{\Delta_{x}}$ on $\Delta_{x} \subset \Delta_{Q}$, where $q=r e^{i \theta}$. Therefore

$$
\int_{W_{\varepsilon}}(F, F)_{k}^{\beta} \operatorname{vol}_{\mathcal{D}}=C \cdot \int_{\varepsilon}^{R} r^{-1} d r \int_{0}^{2 \pi} d \theta \int_{\Delta_{x}}|F(\Omega)|^{2 \beta} \cdot \operatorname{det}(\operatorname{Im} \Omega)^{k \beta-g-1} \operatorname{vol}_{\Delta_{x}} .
$$

Since $F(\Omega)=O(1)$ as $r=|q| \rightarrow 0$, Lemma 5.1 implies that

$$
\begin{aligned}
\int_{W_{\varepsilon}}(F, F)_{k}^{\beta} \operatorname{vol}_{\mathcal{D}} & \leq C \cdot \int_{\varepsilon}^{R} r^{-1} d r \int_{0}^{2 \pi} d \theta \int_{\Delta_{x}}|\log r|^{g^{\prime}(k \beta-g-1)} \operatorname{vol}_{\Delta_{x}} \\
& =C \cdot \int_{\varepsilon}^{R}|\log r|^{g^{\prime(k \beta-g-1)}} r^{-1} d r
\end{aligned}
$$

where $C>0$ are some constants independent of $\varepsilon$ and $g^{\prime}$ is the rank of $Q$. We have $\log r=o\left(r^{-\alpha^{\prime}}\right)$ for any $\alpha^{\prime}>0$ as $r \rightarrow 0$. Hence

$$
|\log r|^{g^{\prime}(k \beta-g-1)} r^{-1}=o\left(r^{-1-\alpha}\right) \quad(r \rightarrow 0)
$$

for any $\alpha>0$. It follows that

$$
\int_{\varepsilon}^{R}|\log r|^{g^{\prime}(k \beta-g-1)} r^{-1} d r=o\left(\varepsilon^{-\alpha}\right) \quad(\varepsilon \rightarrow 0),
$$

which proves the first assertion.

When $\left.F\right|_{\Delta_{Q}} \not \equiv 0$, this calculation also shows that

$$
\int_{W_{\varepsilon}}(F, F)_{k}^{\beta} \operatorname{vol}_{\mathcal{D}} \geq C^{\prime} \cdot \int_{\varepsilon}^{R}|\log r|^{g^{\prime}(k \beta-g-1)} r^{-1} d r+(\text { const })
$$


for some $C^{\prime}>0$ independent of $\varepsilon \ll R$. When $k \beta \geq g+1$, the right hand side diverges as $\varepsilon \rightarrow 0$. On the other hand, when $\left.F\right|_{\Delta_{Q}} \equiv 0$, we have $|F(\Omega)|^{2 \beta}=O\left(r^{2 \beta}\right)$ and so

$$
\int_{W_{\varepsilon}}(F, F)_{k}^{\beta} \operatorname{vol}_{\mathcal{D}} \leq C \cdot \int_{\varepsilon}^{R}|\log r|^{g^{\prime}(k \beta-g-1)} r^{-1+2 \beta} d r \leq C \cdot \int_{\varepsilon}^{R} r^{\delta} d r
$$

for some $\delta>-1$. Therefore $\int_{W_{\varepsilon}}(F, F)_{k}^{\beta} \operatorname{vol}_{\mathcal{D}}$ converges in this case.

Note that the "only if" direction in the second assertion holds with no restriction on $k \beta$.

\section{6. $L^{2 / m}$ CRITERION}

This section is independent of the previous sections. We prepare a general criterion for the pole order of a pluricanonical form in terms of the asymptotic behavior of its integral. This will be used in $\$ 8$ and $\$ 9$.

6.1. $L^{2 / m}$ norm of $m$-canonical forms. Let $U$ be a complex manifold of dimension $N$, and $\omega$ a (holomorphic) $m$-canonical form on $U$. We define the $L^{2 / m}$ norm of $\omega$ as follows. Let $\bar{\omega}$ be the complex conjugate of $\omega$. After a constant multiple, $\omega \wedge \bar{\omega}$ gives a real, nonnegative $C^{\infty}$ section of the real line bundle $\left(\bigwedge^{2 N} \Omega_{U, \mathbb{R}}\right)^{\otimes m}$, where $\Omega_{U, \mathbb{R}}$ is the real cotangent bundle of $U$. To be more precise, if we locally write $\omega=f(z)\left(d z_{1} \wedge \cdots \wedge d z_{N}\right)^{\otimes m}$ with $z_{\alpha}=x_{\alpha}+i y_{\alpha}$, then

$$
\begin{aligned}
\omega \wedge \bar{\omega} & =|f(z)|^{2}\left(d z_{1} \wedge \cdots \wedge d z_{N} \wedge d \bar{z}_{1} \wedge \cdots \wedge d \bar{z}_{N}\right)^{\otimes m} \\
& =i^{N(N-2) m}|f(z)|^{2}\left(d x_{1} \wedge d y_{1} \wedge \cdots \wedge d x_{N} \wedge d y_{N}\right)^{\otimes m} .
\end{aligned}
$$

Globally, if we choose a volume form $\operatorname{vol}_{U}$ on $U$, we can write

$$
\omega \wedge \bar{\omega}=i^{N(N-2) m} \varphi(z) \operatorname{vol}_{U}^{\otimes m}
$$

for some real, nonnegative $C^{\infty}$ function $\varphi(z)$ on $U$. We put $\|\omega\|^{2}=\varphi(z) \operatorname{vol}_{U}^{\otimes m}$ and define its $m$-th root by

$$
\|\omega\|^{2 / m}=\sqrt[m]{\varphi(z)} \operatorname{vol}_{U}
$$

Then $\|\omega\|^{2 / m}$ is a real, nonnegative, continuous $(N, N)$ form on $U$ which is $C^{\infty}$ outside the zero divisor of $\omega$. This definition does not depend on the choice of $\operatorname{vol}_{U}$. The integral $\int_{U}\|\omega\|^{2 / m}$ is the norm we want to look at.

6.2. Criterion for pole order. Now let $X$ be a complex manifold and $\Delta \subset$ $X$ be a smooth irreducible divisor. We take a normal parameter of $\Delta$ and denote by $T_{r}$ the tubular neighborhood of $\Delta$ of radius $r$. We fix a sufficiently small $0<R \ll 1$. For $0<\varepsilon<R$ we set $U_{\varepsilon}=T_{R}-T_{\varepsilon}$, which is the annulus bundle of radius $[\varepsilon, R]$ around $\Delta$. Let $\omega$ be an $m$-canonical form on 
$X-\Delta$. Our purpose is to relate the pole order of $\omega$ along $\Delta$ to the asymptotic behavior of the integral

$$
\int_{U_{\varepsilon}}\|\omega\|^{2 / m} \quad(\varepsilon \rightarrow 0)
$$

Since the problem is local, we shall assume that $X$ is a polydisc in $\mathbb{C}^{N}$, with coordinate $\left(z_{1}, \cdots, z_{N}\right), \Delta$ is defined by $z_{1}=0$, and $T_{r}$ is given by $\left|z_{1}\right|<r$. Then $U_{\varepsilon}$ is defined by $\varepsilon \leq\left|z_{1}\right| \leq R$. We can express $\omega=f(z)\left(d z_{1} \wedge\right.$ $\left.\cdots \wedge d z_{N}\right)^{\otimes m}$ for a holomorphic function $f(z)$ on $X-\Delta$. Then

$$
\begin{aligned}
\|\omega\|^{2 / m} & =|f(z)|^{2 / m} d x_{1} \wedge d y_{1} \wedge \cdots \wedge d x_{N} \wedge d y_{N} \\
& =|f(z)|^{2 / m} r d r \wedge d \theta \wedge d x_{2} \wedge d y_{2} \wedge \cdots \wedge d x_{N} \wedge d y_{N}
\end{aligned}
$$

where $z_{\alpha}=x_{\alpha}+i y_{\alpha}$ and $z_{1}=r e^{i \theta}$. So its integral over $U_{\varepsilon}$ is expressed as

$$
\int_{U_{\varepsilon}}\|\omega\|^{2 / m}=\int_{\varepsilon}^{R} r d r \int_{0}^{2 \pi} d \theta \int_{\Delta}|f(z)|^{2 / m} d x_{2} \wedge \cdots \wedge d y_{N} .
$$

The function $f(z)$ is meromorphic over $X$ if and only if we can write $f(z)=$ $g(z) / z_{1}^{v}$ for some $v \in \mathbb{Z}$ and a holomorphic function $g(z)$ on $X$ such that $\left.g\right|_{\Delta} \not \equiv 0$. This $v$ is the pole order of $f$ (and of $\omega$ ) along $\Delta$.

Proposition 6.1. Let $v$ be the pole order of $\omega$ along $\Delta$.

(1) We have $v \leq m$ if and only if $\int_{U_{\varepsilon}}\|\omega\|^{2 / m}=o\left(\varepsilon^{-2 / m}\right)$.

(2) We have $v \leq m-1$ if and only if $\int_{U_{\varepsilon}}\|\omega\|^{2 / m}=O(1)$.

Proof. If $f(z)$ is not meromorphic over $X$, then for any $a>0,|f(z)|^{2}$ diverges faster than $\left|z_{1}\right|^{-a}$ along an open subset of $\Delta$. Then $\int_{U_{\varepsilon}}\|\omega\|^{2 / m}$ diverges faster than $\varepsilon^{-b}$ for any $b>0$. So we may assume that $f(z)$ is meromorphic over $X$ and write $f(z)=g(z) / z_{1}^{v}$ where $g(z)$ is holomorphic over $X$ with $\left.g\right|_{\Delta} \not \equiv 0$, and $v$ is the pole order of $\omega$ along $\Delta$.

By (6.1), we have

$$
\int_{U_{\varepsilon}}\|\omega\|^{2 / m}=\int_{\varepsilon}^{R} r^{1-2 v / m} d r \int_{0}^{2 \pi} d \theta \int_{\Delta}|g(z)|^{2 / m} d x_{2} \wedge \cdots \wedge d y_{N} .
$$

As a function of $r$, the integral

$$
\int_{0}^{2 \pi} d \theta \int_{\Delta}|g(z)|^{2 / m} d x_{2} \wedge \cdots \wedge d y_{N}
$$

is continuous at $0 \leq r \leq R$, and has a nonzero value at $r=0$ by $\left.g\right|_{\Delta} \not \equiv 0$. Therefore

$$
\int_{0}^{2 \pi} d \theta \int_{\Delta}|g(z)|^{2 / m} d x_{2} \wedge \cdots \wedge d y_{N}=C+o(1) \quad(r \rightarrow 0)
$$


for some constant $C>0$. It follows that

$$
\int_{U_{\varepsilon}}\|\omega\|^{2 / m}=\int_{\varepsilon}^{R} r^{1-2 v / m}(C+o(1)) d r
$$

When $v<m$, this shows that $\int_{U_{\varepsilon}}\|\omega\|^{2 / m}=O(1)$. When $v \geq m$, we obtain

$C^{\prime}|\log \varepsilon|+($ const $) \leq \int_{U_{\varepsilon}}\|\omega\|^{2 / m} \leq C^{\prime \prime}|\log \varepsilon|+($ const $) \quad$ when $v=m$, $C^{\prime} \varepsilon^{2(1-v / m)}+($ const $) \leq \int_{U_{\varepsilon}}\|\omega\|^{2 / m} \leq C^{\prime \prime} \varepsilon^{2(1-v / m)}+$ (const) $\quad$ when $v>m$, for some constants $C^{\prime}, C^{\prime \prime}>0$ independent of $\varepsilon \ll 1$. This first shows the equivalence in (2). The smallest $v$ with $v>m$ is $v=m+1$, for which $\varepsilon^{2(1-v / m)}=\varepsilon^{-2 / m}$. This implies the equivalence in (1).

For our argument in $\$ 8$, it is crucial in (1) to pass from the bound $O(\log \varepsilon)$ to the (seemingly) weaker $o\left(\varepsilon^{-2 / m}\right)$, which creates a room for the estimate.

Remark 6.2. This criterion does not depend on the choice of the normal parameter $z_{1}$ for $\Delta$. Indeed, if $z_{1}^{\prime}$ is another normal parameter, there exist constants $c, c^{\prime}>0$ such that $c\left|z_{1}^{\prime}\right| \leq\left|z_{1}\right|$ and $c^{\prime}\left|z_{1}\right| \leq\left|z_{1}^{\prime}\right|$ around $\Delta$. If $T_{r}^{\prime}=$ $\left\{\left|z_{1}^{\prime}\right| \leq r\right\}$ is the tubular neighborhood of radius $r$ with respect to $z_{1}^{\prime}$, then $T_{c^{\prime} r}^{\prime} \subset T_{r}$ and $T_{c r} \subset T_{r}^{\prime}$. Writing $U_{r}^{\prime}=T_{R}^{\prime}-T_{r}^{\prime}$, we have $U_{\varepsilon} \subset U_{c^{\prime} \varepsilon}^{\prime}$ and $U_{\varepsilon}^{\prime} \subset$ $U_{c \varepsilon}$ up to a region independent of $0<\varepsilon \ll 1$. Thus, if $\int_{U_{\varepsilon}}\|\omega\|^{2 / m}=o\left(\varepsilon^{-\alpha}\right)$ holds, then

$$
\int_{U_{\varepsilon}^{\prime}}\|\omega\|^{2 / m} \leq \int_{U_{c \varepsilon}}\|\omega\|^{2 / m}+(\text { const })=o\left(c^{-\alpha} \varepsilon^{-\alpha}\right)=o\left(\varepsilon^{-\alpha}\right),
$$

and vise versa.

We also want to have a simple normal crossing version of (2). Let $X$ be again a polydisc in $\mathbb{C}^{N}$ and let $\Delta$ now be defined by $z_{1} \cdots z_{k}=0$. We take a smaller closed polydisc $V \subset X$.

Proposition 6.3. An m-canonical form $\omega$ on $X-\Delta$ has at most pole of order $m-1$ along every component of $\Delta$ if and only if $\int_{V-\Delta}\|\omega\|^{2 / m}<\infty$.

Proof. The "if" direction follows from Proposition 6.1(2), so we only have to consider the "only if" direction. We can write

$$
\omega=f(z) \cdot\left(z_{1} \cdots z_{k}\right)^{1-m} \cdot\left(d z_{1} \wedge \cdots \wedge d z_{N}\right)^{\otimes m}
$$

for some holomorphic function $f(z)$ on $X$. Writing $z_{\alpha}=r_{\alpha} e^{i \theta_{\alpha}}$, we have $\begin{aligned}\|\omega\|^{2 / m} & =C \cdot|f(z)|^{2 / m}\left(r_{1} \cdots r_{k}\right)^{2 / m-2} d z_{1} \wedge d \bar{z}_{1} \wedge \cdots \wedge d z_{N} \wedge d \bar{z}_{N} \\ & \leq C^{\prime} \cdot\left(r_{1} \cdots r_{k}\right)^{2 / m-1} d r_{1} \wedge d \theta_{1} \wedge \cdots \wedge d r_{k} \wedge d \theta_{k} \wedge d z_{k+1} \wedge \cdots \wedge d \bar{z}_{N} .\end{aligned}$

Since $\int_{\varepsilon}^{1} r^{\delta} d r=O(1)$ if $\delta>-1$, this shows that $\int_{V-\Delta}\|\omega\|^{2 / m}<\infty$. 


\section{Proof of Theorem 1.1}

From now on we begin the proof of our main results stated in $\$ 1$. In this section we prove Theorem 1.1. Most part of this section can be read after $\S 2$. We use the common notation $f: \mathcal{X}^{(n)} \rightarrow \mathcal{D}$ and $f: X^{n}(\Gamma) \rightarrow A(\Gamma)$ for both projections.

7.1. Proof of (1.1). We first derive the isomorphism (1.1) in Theorem 1.1. Recall from $\$ 2.1$ that we have the isomorphism $K_{f} \simeq f^{*} L^{\otimes n}$ over $\mathcal{X}^{(n)}$ and $K_{\mathcal{D}} \simeq L^{\otimes g+1}$ over $\mathcal{D}$. Combining them, we obtain the isomorphism

$$
K_{\mathcal{X}^{(n)}}^{\otimes m} \simeq K_{f}^{\otimes m} \otimes f^{*} K_{\mathcal{D}}^{\otimes m} \simeq f^{*} L^{\otimes(g+n+1) m}
$$

over $\mathcal{X}^{(n)}$.

Let $\Gamma$ be a finite-index subgroup of $\operatorname{Sp}(\Lambda)$. We first consider the case $\Gamma$ is torsion-free. The $\Gamma$-linearized line bundles $K_{\mathcal{X}^{(n)}}, K_{f}, K_{\mathcal{D}}$ on $\mathcal{X}^{(n)}$ and $\mathcal{D}$ descend to the line bundles $K_{X^{n}(\Gamma)}, K_{f}, K_{A(\Gamma)}$ on $X^{n}(\Gamma)$ and $A(\Gamma)$. Also $L$ descends to a line bundle on $A(\Gamma)$ which we again denote by $L$. Since the isomorphism (7.1) is $\Gamma$-equivariant, it descends to the isomorphism

$$
K_{X^{n}(\Gamma)}^{\otimes m} \simeq f^{*} L^{\otimes(g+n+1) m}
$$

of line bundles over $X^{n}(\Gamma)$. Taking global sections over $X^{n}(\Gamma)$ gives

$$
\begin{aligned}
H^{0}\left(X^{n}(\Gamma), K_{X^{n}(\Gamma)}^{\otimes m}\right) & \simeq H^{0}\left(X^{n}(\Gamma), f^{*} L^{\otimes(g+n+1) m}\right) \\
& \simeq H^{0}\left(A(\Gamma), L^{\otimes(g+n+1) m}\right)=M_{(g+n+1) m}(\Gamma) .
\end{aligned}
$$

Clearly this isomorphism is compatible with multiplication, and we obtain the isomorphism (1.1) in this case.

We next consider the general case $\Gamma$ is not necessarily torsion-free.

Lemma 7.1. The projection $\mathcal{X}^{(n)} \rightarrow X^{n}(\Gamma)$ is unramified in codimension 1.

Proof. Let $\gamma \neq \mathrm{id} \in \Gamma$ be an element of finite order. It suffices to show that the fixed locus of $\gamma$ on $\mathcal{X}^{(n)}$ has codimension $\geq 2$. When $\gamma=-\mathrm{id}$, the fixed locus is the sections of order $\leq 2$ points in the abelian fibration $\mathcal{X}^{(n)} \rightarrow \mathcal{D}$, which has codimension $g n \geq 2$. When $\gamma \neq \pm \mathrm{id}, \gamma$ acts on $\mathcal{D}$ nontrivially. If $[V] \in \mathcal{D}$ is a fixed point of $\gamma$, the $\gamma$-action on the linear space $V$ is nontrivial, so $\gamma$ acts on the fiber $\left(V^{\vee} / \Lambda\right)^{n}$ nontrivially. Then the fixed locus of $\gamma$ on $\mathcal{X}^{(n)}$ has codimension $\geq 1+1=2$.

We choose a torsion-free normal subgroup $\Gamma^{\prime} \triangleleft \Gamma$ of finite index. The quotient group $G=\Gamma / \Gamma^{\prime}$ acts on $X^{n}\left(\Gamma^{\prime}\right) \rightarrow A\left(\Gamma^{\prime}\right)$ with quotient $X^{n}(\Gamma) \rightarrow$ $A(\Gamma)$. By the previous step for $\Gamma^{\prime}$, we have an isomorphism

$$
H^{0}\left(X^{n}\left(\Gamma^{\prime}\right), K_{X^{n}\left(\Gamma^{\prime}\right)}^{\otimes m}\right) \simeq M_{(g+n+1) m}\left(\Gamma^{\prime}\right)
$$


which by construction is $G$-equivariant. We take the $G$-invariant part of this isomorphism. For the right side we have by definition

$$
M_{(g+n+1) m}\left(\Gamma^{\prime}\right)^{G}=M_{(g+n+1) m}(\Gamma) .
$$

For the left side, since $X^{n}\left(\Gamma^{\prime}\right) \rightarrow X^{n}(\Gamma)$ is unramified in codimension 1 by Lemma 7.1, we have

$$
H^{0}\left(X^{n}\left(\Gamma^{\prime}\right), K_{X^{n}\left(\Gamma^{\prime}\right)}^{\otimes m}\right)^{G}=H^{0}\left(X^{n}(\Gamma), K_{X^{n}(\Gamma)}^{\otimes m}\right) .
$$

We thus obtain the isomorphism (1.1).

Example 7.2. Let $g, n, m$ be odd and assume $-1 \in \Gamma$. Then there is no nonzero $m$-canonical form on $X^{n}(\Gamma)$. Indeed, $M_{k}(\Gamma)=0$ for $k=(g+n+1) m$ odd, because $-1 \in \Gamma$ acts on $L^{\otimes k}$ by multiplication by $(-1)^{g k}=-1$.

7.2. Proof of (1.2). Next we show that the $m=1$ component of (1.1) gives the isomorphism (1.2). This is a consequence of the equality of the Petersson norm of a modular form and the $L^{2}$ norm of the corresponding canonical form. For later use in $\$ 8$ and $\$ 9$, we formulate this relation for $m$ canonical forms with $m \geq 1$. (In this section we only need the case $m=1$.)

Proposition 7.3. Let $B$ be an open set of $\mathcal{D}$. Let $F$ be a section of $L^{\otimes(g+n+1) m}$ over $B$ and $\omega$ be the $m$-canonical form on $f^{-1}(B) \subset \mathcal{X}^{(n)}$ corresponding to $f^{*} F$ by the isomorphism $K_{\chi^{(n)}}^{\otimes m} \simeq f^{*} L^{\otimes(g+n+1) m}$. Then we have

$$
\int_{f^{-1}(B)}\|\omega\|^{2 / m}=\int_{B}(F, F)_{(g+n+1) m}^{1 / m} \operatorname{vol}_{\mathcal{D}}
$$

up to a constant independent of $F$ and $B$.

Proof. Since the problem is local over $\mathcal{D}$, we may assume that $B$ is sufficiently small. Since $K_{\mathcal{X}^{(n)}}^{\otimes m} \simeq f^{*} K_{\mathcal{D}}^{\otimes m} \otimes K_{f}^{\otimes m}$ and $f_{*} K_{f}$ is invertible, we can write $\omega$ as

$$
\omega=f^{*} \varphi \cdot f^{*} \omega_{B}^{\otimes m} \otimes \omega_{f}^{\otimes m}
$$

where $\varphi$ is a holomorphic function on $B, \omega_{B}$ a nowhere vanishing canonical form on $B$, and $\omega_{f}$ a nowhere vanishing relative canonical form on $f^{-1}(B)$. Then we have

$$
\|\omega\|^{2 / m}=C \cdot f^{*}|\varphi|^{2 / m} \cdot f^{*}\left(\omega_{B} \wedge \bar{\omega}_{B}\right) \otimes\left(\omega_{f} \wedge \bar{\omega}_{f}\right)
$$

and hence

$$
\int_{f^{-1}(B)}\|\omega\|^{2 / m}=C \cdot \int_{B}\left(\int_{f^{-1}(B) / B} \omega_{f} \wedge \bar{\omega}_{f}\right)|\varphi|^{2 / m} \omega_{B} \wedge \bar{\omega}_{B},
$$

where $\int_{f^{-1}(B) / B}$ means fiber integral.

On the other hand, under the isomorphism $K_{f} \simeq f^{*} L^{\otimes n}$ we have $\omega_{f}=$ $f^{*} F_{1}$ for some section $F_{1}$ of $L^{\otimes n}$ over $B$, and under the isomorphism $K_{\mathcal{D}} \simeq$ 
$L^{\otimes g+1}$ we have $\omega_{B}=F_{2}$ for some section $F_{2}$ of $L^{\otimes g+1}$ over $B$. By construction we have $F=\varphi \cdot F_{1}^{\otimes m} \otimes F_{2}^{\otimes m}$, and hence

$$
(F, F)_{(g+n+1) m}=|\varphi|^{2} \cdot\left(F_{1}, F_{1}\right)_{n}^{m} \cdot\left(F_{2}, F_{2}\right)_{g+1}^{m} .
$$

By Lemma 2.1 and (2.2), we see that

$$
\begin{aligned}
\int_{B}(F, F)_{(g+n+1) m}^{1 / m} \operatorname{vol}_{\mathcal{D}} & =\int_{B}|\varphi|^{2 / m} \cdot\left(F_{1}, F_{1}\right)_{n} \cdot\left(F_{2}, F_{2}\right)_{g+1} \operatorname{vol}_{\mathcal{D}} \\
& =\int_{B}\left(\int_{f^{-1}(B) / B} \omega_{f} \wedge \bar{\omega}_{f}\right)|\varphi|^{2 / m} \omega_{B} \wedge \bar{\omega}_{B} \\
& =\int_{f^{-1}(B)}\|\omega\|^{2 / m}
\end{aligned}
$$

up to a constant. This proves Proposition 7.3.

The isomorphism (1.2) is deduced as follows. Let $F \in M_{g+n+1}(\Gamma)$ and $\omega \in H^{0}\left(K_{X^{n}(\Gamma)}\right)$ be the corresponding canonical form. By Proposition 7.3 with $m=1$, we have

$$
\int_{X^{n}(\Gamma)} \omega \wedge \bar{\omega}=\int_{A(\Gamma)}(F, F)_{g+n+1} \operatorname{vol}_{\mathcal{D}} \leq \infty .
$$

As is well-known, $\int_{X^{n}(\Gamma)} \omega \wedge \bar{\omega}$ converges if and only if $\omega$ extends holomorphically over a smooth projective model $X$ of $X^{n}(\Gamma)$ (cf. Proposition 6.1). On the other hand, by Proposition 5.2, $\int_{A(\Gamma)}(F, F)_{g+n+1} \operatorname{vol}_{\mathcal{D}}$ converges if and only if $F$ is a cusp form. Thus $\omega$ extends over $X$ if and only if $F$ is a cusp form.

We give application of the correspondence (1.2) to the Kodaira dimension of $X^{n}(\Gamma)$ in a few cases.

Example 7.4. For $\Gamma=\Gamma_{g}=\operatorname{Sp}(2 g, \mathbb{Z}), A_{g, n}=X^{n}\left(\Gamma_{g}\right)$ is the moduli space of $n$-pointed principally polarized abelian varieties of genus $g$. When $g \leq 5$, $A_{g, 1}$ is unirational ([33], [4]). On the other hand, when $g \leq 6$, we have the following knowledge about cusp forms.

(1) When $g=2$, by Igusa [10], we have a unique cusp form of weight 10 , which is the minimal weight. Hence $A_{2,7}$ has geometric genus 1 and $\kappa\left(A_{2, n}\right) \geq 0$ for $n \geq 7$.

(2) When $g=3$, by Tsuyumine ([31] Corollary in $\S 20)$, we have a unique cusp form of weight 12 , which is the minimal weight. Hence $A_{3,8}$ has geometric genus 1 and $\kappa\left(A_{3, n}\right) \geq 0$ for $n \geq 8$.

(3) When $g=4$, we have the Schottky form as a cusp form of weight 8 ([11]). By Salvati-Manni [25], this is the minimal weight and $\operatorname{dim} S_{8}\left(\Gamma_{4}\right)=$ 1. Hence $A_{4,3}$ has geometric genus 1 and $\kappa\left(A_{4, n}\right) \geq 0$ for $n \geq 3$. $A_{4,2}$ is the only missing case in $g=4$. 
(4) When $g=5$, we have $\operatorname{dim} S_{12}\left(\Gamma_{5}\right)=2$ by Neeb-Venkov [21], and this is the minimal weight by Poor-Yuen [23]. Hence $A_{5,6}$ has geometric genus 2 and $\kappa\left(A_{5, n}\right)>0$ for $n \geq 6$.

(5) When $g=6$, we have $\operatorname{dim} S_{12}\left(\Gamma_{6}\right) \geq 3$ by Neeb-Venkov [21]. Hence $A_{6,5}$ has geometric genus $\geq 3$ and $\kappa\left(A_{6, n}\right)>0$ for $n \geq 5$. Poor-Yuen [23] showed that there is no cusp form of weight $\leq 8$.

7.3. Vector-valued Siegel modular forms. This subsection is a sort of appendix to $\$ 7.1$. We combine a generalization of the argument in $\$ 7.1$ with the Leray spectral sequence for $X^{n}(\Gamma) \rightarrow A(\Gamma)$. This gives a spectral sequence that relates the cohomology of $K_{X^{n}(\Gamma)}^{\otimes m}$ to the cohomology of certain vector bundles on $A(\Gamma)$ whose sections are vector-valued modular forms. The result of this subsection will not be used in other sections.

We assume that $\Gamma$ is torsion-free. The universal quotient bundle $F$ over $\mathcal{D}$ descends to a vector bundle over $A(\Gamma)$ which we again denote by $F$.

Proposition 7.5. Assume that $\Gamma$ is torsion-free. For each $m \geq 0$ there exists a spectral sequence

$$
E_{2}^{p, q}=H^{p}\left(A(\Gamma), \bigwedge^{q}\left(F^{\oplus n}\right) \otimes L^{\otimes(g+n+1) m}\right) \Rightarrow H^{p+q}\left(X^{n}(\Gamma), K_{X^{n}(\Gamma)}^{\otimes m}\right) .
$$

Proof. We abbreviate $X=X^{n}(\Gamma)$. We shall rewrite the $E_{2}$ page of the Leray spectral sequence

$$
E_{2}^{p, q}=H^{p}\left(A(\Gamma), R^{q} f_{*}\left(K_{X}^{\otimes m}\right)\right) \Rightarrow H^{p+q}\left(X, K_{X}^{\otimes m}\right) .
$$

Since $K_{X} \simeq f^{*} L^{\otimes g+n+1}$, we have

$$
R^{q} f_{*}\left(K_{X}^{\otimes m}\right) \simeq R^{q} f_{*} f^{*} L^{\otimes(g+n+1) m} \simeq R^{q} f_{*} O_{X} \otimes L^{\otimes(g+n+1) m}
$$

by the projection formula. We shall show that

$$
R^{q} f_{*} O_{X} \simeq \bigwedge^{q}\left(F^{\oplus n}\right)
$$

By Grauert's theorem ([7] III.12.9), $R^{q} f_{*} O_{X}$ is locally free and its fiber over a point $[A]=\left[V^{\vee} / \Lambda\right]$ of $A(\Gamma)$ is identified with $H^{q}\left(O_{A^{n}}\right)$. We have the canonical isomorphisms

$$
H^{q}\left(O_{A^{n}}\right) \simeq H^{0}\left(\Omega_{A^{n}}^{q}\right)^{\vee} \simeq \bigwedge^{q} H^{0}\left(\Omega_{A^{n}}^{1}\right)^{\vee} \simeq \bigwedge^{q}\left(H^{0}\left(\Omega_{A}^{1}\right)^{\vee}\right)^{\oplus n} \simeq \bigwedge^{q}\left(V^{\vee}\right)^{\oplus n}
$$

Here the first isomorphism is induced from the Hodge pairing

$$
H^{q, 0}\left(A^{n}\right) \times H^{0, q}\left(A^{n}\right) \rightarrow \mathbb{C}, \quad(\omega, \eta) \mapsto \int_{A^{n}} \omega \wedge \eta \wedge h^{g n-q},
$$

where $h$ is the polarization on $A^{n}$ induced from the given symplectic form. The space $\bigwedge^{q}\left(V^{\vee}\right)^{\oplus n}$ is the fiber of $\bigwedge^{q}\left(E^{\vee}\right)^{\oplus n} \simeq \bigwedge^{q} F^{\oplus n}$ over $[A]$. 
Sections of $\bigwedge^{q}\left(F^{\oplus n}\right) \otimes L^{\otimes k}$ over $A(\Gamma)$ are identified with modular forms of weight $k$ for $\Gamma$ with values in $\bigwedge^{q}\left(\mathrm{St}^{\oplus n}\right)^{\vee}$ where $\mathrm{St}$ is the standard representation of $\mathrm{GL}_{g}(\mathbb{C})$. Thus the edge morphism of (7.2) at the side $p=0$ takes the form

$$
H^{q}\left(X^{n}(\Gamma), K_{X^{n}(\Gamma)}^{\otimes m}\right) \rightarrow M_{(g+n+1) m}\left(\Gamma, \bigwedge^{q}\left(\mathrm{St}^{\oplus n}\right)^{\vee}\right) .
$$

The edge morphism at the other side $q=0$ takes the form

$$
H^{p}\left(A(\Gamma), L^{\otimes(g+n+1) m}\right) \rightarrow H^{p}\left(X^{n}(\Gamma), K_{X^{n}(\Gamma)}^{\otimes m}\right) .
$$

\section{Proof of Theorem 1.2}

In this section we prove Theorem 1.2. Let us begin with recalling the setting. Let $X$ be a complex analytic variety containing $X^{n}(\Gamma)$ as a Zariski open set. We assume that

- the singular locus of $X$ has codimension $\geq 2$,

- $X^{n}(\Gamma) \rightarrow A(\Gamma)$ extends to a morphism $f: X \rightarrow A(\Gamma)^{\Sigma}$ to some toroidal compactification of $A(\Gamma)$, and

- every irreducible component of $\Delta_{X}=X-X^{n}(\Gamma)$ dominates some irreducible component of $\Delta_{A}=A(\Gamma)^{\Sigma}-A(\Gamma)$.

By the second condition, $\Delta_{X}=f^{-1}\left(\Delta_{A}\right)$ is a divisor of $X$. We want to show that the isomorphism $H^{0}\left(K_{X^{n}(\Gamma)}^{\otimes m}\right) \simeq M_{(g+n+1) m}(\Gamma)$ in $\$ 7.1$ extends to an isomorphism

$$
H^{0}\left(X, K_{X}^{\otimes m}\left(m \Delta_{X}\right)\right) \simeq M_{(g+n+1) m}(\Gamma) .
$$

Since restriction to $X^{n}(\Gamma) \subset X$ gives an inclusion

$$
H^{0}\left(X, K_{X}^{\otimes m}\left(m \Delta_{X}\right)\right) \hookrightarrow H^{0}\left(X^{n}(\Gamma), K_{X^{n}(\Gamma)}^{\otimes m}\right),
$$

it is sufficient to show that this is actually equality. In other words, we want to show that every $m$-canonical form on $X^{n}(\Gamma)$ has at most pole of order $m$ along every irreducible component of $\Delta_{X}$. We deduce this property by applying the $L^{2 / m}$ criterion in $\$$. The estimate of $L^{2 / m}$ norm required there essentially reduces to the asymptotic estimate of Petersson norm in $\$ 5$,

8.1. Pullback to relative torus embedding. In this subsection we translate the $L^{2 / m}$ criterion on $X$ to that on the family over the torus fibration associated to each cusp. Let $I$ be a primitive isotropic sublattice of $\Lambda$. Let $\mathcal{X}_{I}^{(n)}=\mathcal{X}^{(n)} / U(I)_{\mathbb{Z}}$ be the $n$-fold Kuga family over $\mathcal{B}_{I}=\mathcal{D} / U(I)_{\mathbb{Z}}$. The 
situation around the I-cusp is as follows:

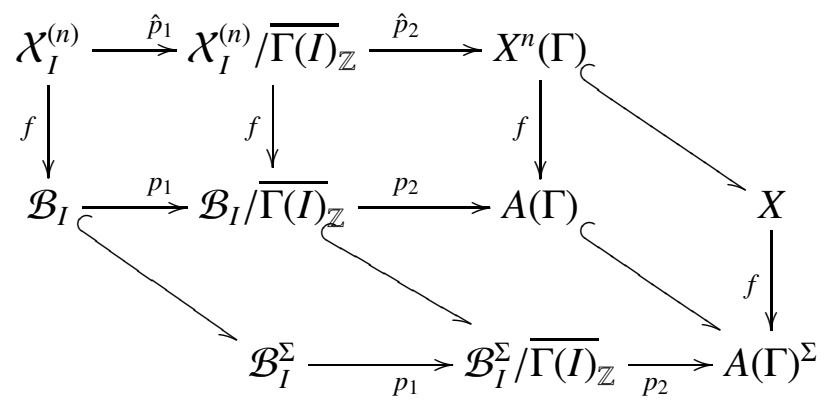

We write $p=p_{2} \circ p_{1}$ and $\hat{p}=\hat{p}_{2} \circ \hat{p}_{1}$.

Let $\mathbb{R}_{\geq 0} Q \subset U(I)_{\mathbb{R}}$ be a positive definite ray in $\Sigma_{I}$, where $Q$ is a primitive vector of $U(I)_{\mathbb{Z}}$. Let $\Delta_{Q}=\Delta_{Q, I}$ be the boundary stratum of $\mathcal{B}_{I}^{\Sigma}$ of codimension 1 corresponding to $\mathbb{R}_{\geq 0} Q$, and we put $\Delta_{Q}^{\prime}=p\left(\Delta_{Q}\right) \subset A(\Gamma)^{\Sigma}$. We write $a$ for the ramification index of $\mathcal{B}_{I}^{\Sigma} \rightarrow A(\Gamma)^{\Sigma}$ at $\Delta_{Q}$. We shall localize the situation. We take a general point $x$ of $\Delta_{Q}$, its small neighborhood $V$ in $\mathcal{B}_{I}^{\Sigma}$, and its small neighborhood $\Delta_{x}$ in $\Delta_{Q}$ contained in $V$. Then $y=p(x)$ is a general point of $\Delta_{Q}^{\prime}, V^{\prime}=p(V)$ is a small neighborhood of $y$ in $A(\Gamma)^{\Sigma}, \Delta_{y}^{\prime}=p\left(\Delta_{x}\right)$ is a small neighborhood of $y$ in $\Delta_{Q}^{\prime}$, and $p: \Delta_{x} \rightarrow \Delta_{y}^{\prime}$ is isomorphic. In some local coordinates around $x$ and $y, p: V \rightarrow V^{\prime}$ is expressed as

$$
\left(z, z_{1}, \cdots, z_{N}\right) \mapsto\left(z^{\prime}=z^{a}, z_{1}, \cdots, z_{N}\right),
$$

with $\Delta_{Q}$ defined by $z=0$ and $\Delta_{Q}^{\prime}$ defined by $z^{\prime}=0$.

Remark 8.1. By Theorem 4.3 (2), $a$ equals to the ramification index of $\mathcal{B}_{I}^{\Sigma} \rightarrow \mathcal{B}_{I}^{\Sigma} / \overline{\Gamma(I)}_{\mathbb{Z}}$ at $\Delta_{Q}$. Let $U(I)_{\mathbb{Z}}^{\star}=U(I)_{\mathbb{Q}} \cap\langle\Gamma,-1\rangle$. Using Proposition 3.4, we can show that $a=1$ if $Q / 2 \notin U(I)_{\mathbb{Z}}^{\star}$ and $a=2$ if $Q / 2 \in U(I)_{\mathbb{Z}}^{\star}$, like the classification of regular/irregular cusps in the case $g=1$. We do not need this precise information for the proof of Theorem 1.2 .

We set $U^{\prime}=f^{-1}\left(V^{\prime}\right) \subset X, V^{\circ}=V \backslash \Delta_{Q} \subset \mathcal{B}_{I}$ and $U^{\circ}=f^{-1}\left(V^{\circ}\right) \subset \mathcal{X}_{I}^{(n)}$. The situation is

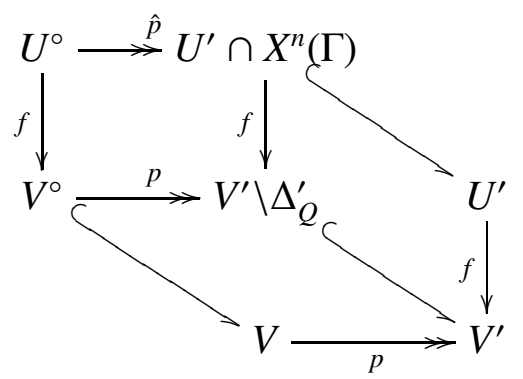

which is a localization of (8.1). Here $U^{\circ} \rightarrow V^{\circ}$ is an abelian fibration, $U^{\prime} \cap X^{n}(\Gamma) \rightarrow V^{\prime} \backslash \Delta_{Q}^{\prime}$ is an abelian or Kummer fibration, $p$ has degree $a$, and $\hat{p}$ has degree $a$ or $2 a$. 
Let $T_{r}$ be the tubular neighborhood of $\Delta_{x}$ of radius $|z|=r$. Then $p\left(T_{r}\right)$ is the tubular neighborhood of $\Delta_{y}^{\prime}$ of radius $\left|z^{\prime}\right|=r^{a}$. We fix a sufficiently small $0<R \ll 1$, and for $0<\varepsilon<R$ we set

$$
\begin{gathered}
V_{\varepsilon}=T_{R}-T_{\varepsilon} \subset V^{\circ}, \\
V_{\varepsilon}^{\prime}=p\left(V_{\varepsilon}\right) \subset V^{\prime} \backslash \Delta_{Q}^{\prime} .
\end{gathered}
$$

Then $V_{\varepsilon}$ is the annulus bundle of radius $\varepsilon \leq|z| \leq R$ around $\Delta_{x}$, and $V_{\varepsilon}^{\prime}$ is the annulus bundle of radius $\varepsilon^{a} \leq\left|z^{\prime}\right| \leq R^{a}$ around $\Delta_{y}^{\prime}$. Let

$$
\begin{gathered}
U_{\varepsilon}=f^{-1}\left(V_{\varepsilon}\right) \subset U^{\circ}, \\
U_{\varepsilon}^{\prime}=f^{-1}\left(V_{\varepsilon}^{\prime}\right) \subset U^{\prime} \cap X^{n}(\Gamma),
\end{gathered}
$$

be the families over these bases. We have as restriction of (8.2)

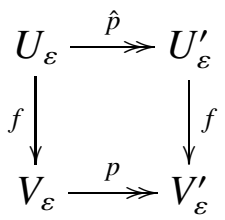

Then $U_{\varepsilon}^{\prime}$ gives an annulus bundle around general point of each component of $\Delta_{X}$ lying over $\Delta_{Q}^{\prime}$. To be more precise, let $f^{-1}\left(\Delta_{Q}^{\prime}\right)=\sum_{i} \Delta_{i}$ be the irreducible decomposition of the reduced divisor $f^{-1}\left(\Delta_{Q}^{\prime}\right) \subset X$. We can write $f^{*} \Delta_{Q}^{\prime}=$ $\sum_{i} d_{i} \Delta_{i}$ for some natural numbers $d_{i}$. By our third assumption of Theorem 1.2, each $\Delta_{i}$ dominates $\Delta_{Q}^{\prime}$, so we can choose a general point $y_{i}$ of $\Delta_{i}$ such that $f\left(y_{i}\right)=y$ and the tangent map $T_{y_{i}} \Delta_{i} \rightarrow T_{y} \Delta_{O}^{\prime}$ is surjective. In some local coordinate around $y_{i}$, the projection $f: X \rightarrow A(\Gamma)^{\Sigma}$ is expressed as

$$
\left(z^{\prime \prime}, z_{1}, \cdots, z_{N}, w_{1}, \cdots, w_{g n}\right) \mapsto\left(z^{\prime}=\left(z^{\prime \prime}\right)^{d_{i}}, z_{1}, \cdots, z_{N}\right),
$$

with $\Delta_{i}$ defined by $z^{\prime \prime}=0$. We take a small neighborhood $\Delta_{y_{i}}$ of $y_{i}$ in $\Delta_{i}$ and let $U_{\varepsilon, i}^{\prime}$ be the restriction of $U_{\varepsilon}^{\prime}$ around $\Delta_{y_{i}}$. Then $U_{\varepsilon, i}^{\prime}$ is the annulus bundle of radius $\varepsilon^{a / d_{i}} \leq\left|z^{\prime \prime}\right| \leq R^{a / d_{i}}$ around $\Delta_{y_{i}}$.

We can now state the version of the $L^{2 / m}$ criterion we will apply.

Lemma 8.2. Let $\omega_{X}$ be an m-canonical form on $U^{\prime} \cap X^{n}(\Gamma)$ and $\omega_{I}$ be the pullback of $\omega_{X}$ to $U^{\circ}$.

(1) Assume that for every positive number $\alpha>0$ the asymptotic estimate

$$
\int_{U_{\varepsilon}}\left\|\omega_{I}\right\|^{2 / m}=o\left(\varepsilon^{-\alpha}\right) \quad(\varepsilon \rightarrow 0)
$$

holds. Then $\omega_{X}$ has at most pole of order $m$ along $U^{\prime} \cap \Delta_{i}$ for every irreducible component $\Delta_{i}$ of $f^{-1}\left(\Delta_{Q}^{\prime}\right)$.

(2) If $\int_{U_{\varepsilon}}\left\|\omega_{I}\right\|^{2 / m}=O(1)$, then $\omega_{X}$ has at most pole of order $m-1$ along $U^{\prime} \cap \Delta_{i}$ for every $\Delta_{i}$. 
Proof. (1) It is sufficient to look at the pole order of $\omega_{X}$ around $\Delta_{y_{i}}$. What has to be shown is that the asymptotic estimate (8.3) for $\omega_{I}$ implies an asymptotic estimate for $\omega_{X}$ around $\Delta_{y_{i}}$ as in Proposition 6.1(1). Since $U_{\varepsilon, i}^{\prime}$ is the annulus bundle of radius $\left[\varepsilon^{a / d_{i}}, R^{a / d_{i}}\right]$ around $\Delta_{y_{i}}$, the estimate in Proposition 6.1 (1) for $\omega_{X}$ is equivalent to the estimate

$$
\int_{U_{\varepsilon, i}^{\prime}}\left\|\omega_{X}\right\|^{2 / m}=o\left(\left(\varepsilon^{a / d_{i}}\right)^{-2 / m}\right)=o\left(\varepsilon^{-2 a / m d_{i}}\right) \quad(\varepsilon \rightarrow 0) .
$$

Thus it suffices to show that (8.3) implies (8.4).

Since $U_{\varepsilon, i}^{\prime} \subset U_{\varepsilon}^{\prime}$ and $\left\|\omega_{X}\right\|^{2 / m}$ is a nonnegative multiple of a volume form, we have

$$
\int_{U_{\varepsilon, i}^{\prime}}\left\|\omega_{X}\right\|^{2 / m} \leq \int_{U_{\varepsilon}^{\prime}}\left\|\omega_{X}\right\|^{2 / m}
$$

Pulling back $\omega_{X}$ to $U_{\varepsilon}$ by $U_{\varepsilon} \rightarrow U_{\varepsilon}^{\prime}$, we have

$$
\int_{U_{\varepsilon}^{\prime}}\left\|\omega_{X}\right\|^{2 / m} \leq \int_{U_{\varepsilon}}\left\|\omega_{I}\right\|^{2 / m} .
$$

Then, if we substitute $\alpha=2 a / m d_{i}$ into $(8.3)$, we obtain

$$
\int_{U_{\varepsilon}}\left\|\omega_{I}\right\|^{2 / m}=o\left(\varepsilon^{-2 a / m d_{i}}\right)
$$

This gives (8.4).

The proof of (2) is similar, using Proposition 6.1(2) in place of Proposition 6.1(1).

8.2. Completion of the proof. We translate the criterion in Lemma 8.2 to that for the corresponding local modular forms. Combining the results so far, we obtain the following.

Proposition 8.3. Let $\omega_{X}$ and $\omega_{I}$ be as in Lemma 8.2 and $k=(g+n+1) m$. Let $F$ be the section of $L^{\otimes k}$ over $V^{\circ}$ such that $\omega_{I}=f^{*} F$ under the isomorphism $K_{X_{I}^{(n)}}^{\otimes m} \simeq f^{*} L^{\otimes k}$ of line bundles over $U^{\circ}$.

(1) If $F$ extends to a holomorphic section of the extended line bundle $L^{\otimes k}$ over $V \subset \mathcal{B}_{I}^{\Sigma}(c f . \$ 4.4)$, then $\omega_{X}$ has at most pole of order $m$ along $U^{\prime} \cap \Delta_{i}$ for every irreducible component $\Delta_{i}$ of $f^{-1}\left(\Delta_{Q}^{\prime}\right)$.

(2) If furthermore $F$ vanishes at $\Delta_{Q}$, then $\omega_{X}$ has at most pole of order $m-1$ along $U^{\prime} \cap \Delta_{i}$ for every $\Delta_{i}$.

Proof. (1) We shall show that the estimate (8.3) holds, which by Proposition 7.3 is equivalent to the estimate

$$
\int_{V_{\varepsilon}}(F, F)_{k}^{1 / m} \operatorname{vol}_{\mathcal{D}}=o\left(\varepsilon^{-\alpha}\right)
$$


We reduce this to Proposition 5.2 by passing from the $I$-cusp to an adjacent 0-dimensional cusp. Choose a maximal isotropic sublattice $J$ of $\Lambda$ containing $I$. By Lemma 4.1, the projection $\mathcal{B}_{I} \rightarrow \mathcal{B}_{J}$ extends to an etale map $\pi: \mathcal{B}_{I}^{\Sigma} \rightarrow \mathcal{B}_{J}^{\Sigma}$. We choose $\chi \in U(J)_{\mathbb{Z}}^{\vee}$ such that $\chi(Q)=1$ and put $q=\exp (2 \pi i \chi(\cdot))$ on $\mathcal{B}_{J} \subset T_{J}$. Let $W_{\varepsilon} \subset \mathcal{B}_{J}$ be the annulus bundle around $\pi\left(\Delta_{x}\right)$ of radius $\varepsilon \leq|q| \leq R$. As explained in Remark 6.2, the asymptotic behavior of the integral over $V_{\varepsilon} \simeq \pi\left(V_{\varepsilon}\right)$ is equivalent to that over $W_{\varepsilon}$. Now we have

$$
\int_{W_{\varepsilon}}(F, F)_{k}^{1 / m} \operatorname{vol}_{\mathcal{D}}=o\left(\varepsilon^{-\alpha}\right)
$$

by the first part of Proposition 5.2 with $\beta=1 / m$, which implies (8.5).

(2) Similarly, we are reduced to showing that

$$
\int_{V_{\varepsilon}}(F, F)_{k}^{1 / m} \operatorname{vol}_{\mathcal{D}}=O(1)
$$

which follows from the second part of Proposition 5.2.

We can now prove Theorem 1.2. We first derive the isomorphism (1.3). Let $\omega_{X}$ be an $m$-canonical form on $X^{n}(\Gamma)$ and $F$ be the corresponding (global) modular form. Then $\omega_{I}$ corresponds to the restriction of $F$ to $V^{\circ}$. By Lemma 4.6, $F$ extends holomorphically over $V$, so we can apply Proposition 8.3 (1). We thus obtain the isomorphism (1.3). The assertion (1.4) for cusp forms follows from the second part of Lemma 4.6 and Proposition 8.3 (2).

It remains to prove the last assertion of Theorem 1.2. Let us recall the definition of klt singularities ([13] §2.3).

Definition 8.4. Let $X$ be a normal complex analytic variety and $\Delta$ be an effective $\mathbb{Q}$-Weil divisor such that $\lfloor\Delta\rfloor=0$. The pair $(X, \Delta)$ is called Kawamata log terminal if $K_{X}+\Delta$ is $\mathbb{Q}$-Cartier and for some (hence all) log resolution $\pi: Y \rightarrow X$ of $(X, \Delta)$, we have $K_{Y}=\pi^{*}\left(K_{X}+\Delta\right)+\sum_{i} a\left(E_{i}\right) E_{i}$ with $a\left(E_{i}\right)>-1$. If $\omega$ is a meromorphic $m$-canonical form on the regular locus of $X$ whose pole divisor satisfies $\leq m \Delta$, its pullback to $Y$ has at most pole of order $m-1$ along every component of the exceptional divisor. (This is the only property where we need the klt condition.)

Now let $X \supset X^{n}(\Gamma)$ be as before and assume that $X$ is normal and compact. We write $\Delta_{U^{\prime}}=f^{-1}\left(\Delta_{Q}^{\prime}\right) \cap U^{\prime}$ for a general point $y$ of an irreducible component $\Delta_{Q}^{\prime}$ of $\Delta_{A}$. What has to be shown is that if $\left(U^{\prime},\left(1-m^{-1}\right) \Delta_{U^{\prime}}\right)$ is klt for every component of $\Delta_{A}$, the map

$$
S_{(g+n+1) m}(\Gamma) \hookrightarrow H^{0}\left(K_{X}^{\otimes m}\left((m-1) \Delta_{X}\right)\right)
$$

is surjective. Let $\omega_{X}$ be an element of $H^{0}\left(K_{X}^{\otimes m}\left((m-1) \Delta_{X}\right)\right)$ and $F$ be the corresponding modular form of weight $k=(g+n+1) m$. In view of Lemma 
4.6, we want to show that $F$ vanishes at $\Delta_{Q, I}$ for every $I$ and positive definite $\mathbb{R}_{\geq 0} Q \in \Sigma_{I}$. If we choose a maximal $J \supset I$, this is equivalent to the vanishing of $F$ at $\Delta_{Q, J}$. By the second part of Proposition 5.2 with $\beta=1 / \mathrm{m}$ (note that $\mathrm{km}^{-1} \geq g+1$ ), it suffices to show that $\int_{W_{\varepsilon}}(F, F)_{k}^{1 / m} \operatorname{vol}_{\mathcal{D}}=O(1)$. Going back by the etale gluing $\mathcal{B}_{I}^{\Sigma_{I}} \rightarrow \mathcal{B}_{J}^{\Sigma_{J}}$, we are reduced to showing that $\int_{V^{\circ}}(F, F)_{k}^{1 / m} \operatorname{vol}_{\mathcal{D}}<\infty$. By Proposition 7.3 this is translated to $\int_{U^{\circ}}\left\|\omega_{I}\right\|^{2 / m}<\infty$, which in turn is equivalent to $\int_{U^{\prime}-\Delta_{U^{\prime}}}\left\|\omega_{X}\right\|^{2 / m}<\infty$.

We take a $\log$ resolution $\left(U^{\prime \prime}, \Delta_{U^{\prime \prime}}\right)$ of $\left(U^{\prime}, \Delta_{U^{\prime}}\right)$ and let $E$ be its exceptional divisor. Then the above condition is rewritten as

$$
\int_{U^{\prime \prime}-E-\Delta_{U^{\prime \prime}}}\left\|\omega_{X}\right\|^{2 / m}<\infty .
$$

The divisor $E+\Delta_{U^{\prime \prime}}$ is simple normal crossing, and can be covered by finitely many local charts of $U^{\prime \prime}$ by the properness of $X \rightarrow A(\Gamma)^{\Sigma}$. Since the pole divisor of $\omega_{X}$ satisfies $\leq m\left(1-m^{-1}\right) \Delta_{U^{\prime}}$ by our assumption on $\omega_{X}$, the klt condition for $\left(U^{\prime},\left(1-m^{-1}\right) \Delta_{U^{\prime}}\right)$ implies that $\omega_{X}$ has at most pole of order $m-1$ along every component of $E+\Delta_{U^{\prime \prime}}$. Then the assertion (8.6) follows from Proposition 6.3. This completes the proof Theorem 1.2.

Remark 8.5. When $U^{\prime}$ is smooth and $\Delta_{U^{\prime}}$ is simple normal crossing, the pair $\left(U^{\prime},\left(1-m^{-1}\right) \Delta_{U^{\prime}}\right)$ is always klt. Hence $S_{(g+n+1) m}(\Gamma)$ is isomorphic to $H^{0}\left(K_{X}^{\otimes m}\left((m-1) \Delta_{X}\right)\right)$ for every $m$ when $X$ is smooth and $\Delta_{X}$ is simple normal crossing over general points of $\Delta_{A}$.

\section{Proof of Theorem 1.3}

In this section we prove Theorem 1.3. In $\$ 9.1$ we prove the assertion (1). In $\$ 9.2$ we prove the assertion (2). For a $\mathbb{Q}$-Weil divisor $D$ on a normal compact complex analytic variety $X$, its litaka dimension $\kappa(D)=\kappa(X, D)$ is defined as the maximum of the dimension of the image of the rational map $\phi_{|m D|}: X \rightarrow \mathbb{P}^{N}$ as $m$ runs ([12], [19]). We write $K_{X}$ for the canonical divisor of $X$ as a Weil divisor or the corresponding rank 1 reflexive sheaf on $X$.

9.1. Canonical map. The canonical map of a smooth projective model of $X^{n}(\Gamma)$ is described as follows.

Proposition 9.1. Let $X$ be a smooth projective model of $X^{n}(\Gamma)$. The canonical map of $X$ factors through

$$
X \sim X^{n}(\Gamma) \rightarrow A(\Gamma) \rightarrow \mathbb{P}^{N}
$$

where $A(\Gamma) \rightarrow \mathbb{P}^{N}$ is the rational map defined by $S_{g+n+1}(\Gamma)$.

When $A(\Gamma) \rightarrow \mathbb{P}^{N}$ is generically finite, this shows that the image of the canonical map of $X$ has dimension $g(g+1) / 2$, which proves the assertion (1) of Theorem 1.3 . 
(Proof of Proposition 9.1). Recall that the isomorphism $S_{g+n+1}(\Gamma) \simeq$ $H^{0}\left(K_{X}\right)$ is explicitly given as follows. We choose a maximal isotropic sublattice $J \subset \Lambda$ and let $s_{J}$ be the associated frame of $L$. Let $\omega_{\mathcal{D}}$ be the canonical form on $\mathcal{D}$ corresponding to $s_{J}^{\otimes g+1}$ by $K_{\mathcal{D}} \simeq L^{\otimes g+1}$, and $\omega_{f}$ be the relative canonical form on $f: \mathcal{X}^{(n)} \rightarrow \mathcal{D}$ corresponding to $f^{*} s_{J}^{\otimes n}$ by $K_{f} \simeq f^{*} L^{\otimes n}$. We put $\omega_{0}=\omega_{f} \wedge f^{*} \omega_{\mathcal{D}}$. If $F s_{J}^{\otimes g+n+1}$ is a cusp form of weight $g+n+1$, the pullback of the corresponding canonical form on $X$ to $\mathcal{X}^{(n)}$ is given by $\left(f^{*} F\right) \omega_{0}$. Thus, if $F_{i} s_{J}^{\otimes g+n+1}, 0 \leq i \leq N$, are basis of $S_{g+n+1}(\Gamma)$, then $\left(f^{*} F_{i}\right) \omega_{0}$ are pullback of the corresponding basis of $H^{0}\left(K_{X}\right)$ to $\mathcal{X}^{(n)}$. This shows that the canonical map of $X$ is given by $\left[f^{*} F_{0}: \cdots: f^{*} F_{N}\right]: X \rightarrow \mathbb{P}^{N}$. This factors through $X \stackrel{f}{f} A(\Gamma) \rightarrow \mathbb{P}^{N}$, where $A(\Gamma) \rightarrow \mathbb{P}^{N}$ is the rational map defined by $\left[F_{0}: \cdots: F_{N}\right]$.

9.2. Proof of (1.5). Next we prove the assertion (2) of Theorem 1.3, Let $X \supset X^{n}(\Gamma)$ be a normal complex analytic variety satisfying the conditions of Theorem 1.2, and $f: X \rightarrow A(\Gamma)^{\Sigma}$ the extended morphism. We choose and fix a natural number $m$ such that $L^{\otimes m}$ can be defined as a line bundle over $A(\Gamma)^{\Sigma}$ in the sense of the last paragraph of $\$ 4.4$. Since $K_{\mathcal{X}^{(n)}}^{\otimes m} \simeq f^{*} L^{\otimes k}$ over $\mathcal{X}^{(n)}$ where $k=(g+n+1) m$, then $K_{\mathcal{X}^{(n)}}^{\otimes m}$ also descends to a line bundle over $X^{n}(\Gamma)$ which we denote by $K_{X^{n}(\Gamma)}^{\otimes m}$ (by abuse of notation). Since $\mathcal{X}^{(n)} \rightarrow$ $X^{n}(\Gamma)$ is unramified in codimension 1 by Lemma 7.1, restriction of $K_{X^{n}(\Gamma)}^{\otimes m}$ to the regular locus of $X^{n}(\Gamma)$ is indeed isomorphic to the $m$-power of its canonical bundle. The isomorphism $K_{\mathcal{X}^{(n)}}^{\otimes m} \simeq f^{*} L^{\otimes k}$ over $\mathcal{X}^{(n)}$ descends to an isomorphism $\left.K_{X^{n}(\Gamma)}^{\otimes m} \simeq f^{*} L^{\otimes k}\right|_{X^{n}(\Gamma)}$ of line bundles over $X^{n}(\Gamma)$.

Proposition 9.2. Let $X, m$ and $k=(g+n+1) m$ be as above. Then the isomorphism $\left.f^{*} L^{\otimes k}\right|_{X^{n}(\Gamma)} \simeq K_{X^{n}(\Gamma)}^{\otimes m}$ of line bundles over $X^{n}(\Gamma)$ extends to an injective homomorphism $f^{*} L^{\otimes k} \hookrightarrow K_{X}^{\otimes m}\left(m \Delta_{X}\right)$ of sheaves on $X$. In particular, we have $m K_{X} \geq f^{*}\left(k L-m \Delta_{A}\right)$.

Proof. We keep the notation of $\$ 8$. Let $y$ be a general point of an irreducible component $\Delta_{Q}^{\prime}$ of $\Delta_{A}$. Let $F$ be a local frame of $L^{\otimes k}$ on a small neighborhood $V^{\prime}$ of $y$ in $A(\Gamma)^{\Sigma}$. Then $f^{*} F$ is a local frame of $f^{*} L^{\otimes k}$ on the neighborhood $U^{\prime}=f^{-1}\left(V^{\prime}\right)$ of $f^{-1}(y)$ in $X$. Let $\omega$ be the $m$-canonical form on $U^{\prime} \cap X^{n}(\Gamma)$ corresponding to the restriction of $f^{*} F$ to $U^{\prime} \cap X^{n}(\Gamma)$ via $\left.K_{X^{n}(\Gamma)}^{\otimes m} \simeq f^{*} L^{\otimes k}\right|_{X^{n}(\Gamma)}$. By Proposition 8.3 (1), $\omega$ has at most pole of order $m$ along $U^{\prime} \cap \Delta_{i}$ for every irreducible component $\Delta_{i}$ of $f^{-1}\left(\Delta_{Q}^{\prime}\right)$. This shows that the isomorphism $\left.f^{*} L^{\otimes k}\right|_{X^{n}(\Gamma)} \simeq K_{X^{n}(\Gamma)}^{\otimes m}$ at $U^{\prime} \cap X^{n}(\Gamma)$ extends to a sheaf homomorphism $\left.f^{*} L^{\otimes k}\right|_{U^{\prime}} \hookrightarrow K_{U^{\prime}}^{\otimes m}\left(m \Delta_{U^{\prime}}\right)$ over $U^{\prime}$. Since we obtain this for general points of every component of $\Delta_{A}$, our condition on $X \rightarrow A(\Gamma)^{\Sigma}$ ensures that we obtain a homomorphism $f^{*} L^{\otimes k} \hookrightarrow K_{X}^{\otimes m}\left(m \Delta_{X}\right)$ outside a codimension $\geq 2$ locus in $X$. By the normality of $X$, this extends over the whole $X$. 
As for the second assertion, we have $m K_{X}+m \Delta_{X} \geq f^{*}(k L)$ by the first assertion. By our condition on $X \rightarrow A(\Gamma)^{\Sigma}$, we can pullback $\Delta_{A}$ to a Weil divisor of $X$ whose support is $\Delta_{X}$. (Pullback $\Delta_{A} \cap A(\Gamma)_{\text {reg }}^{\Sigma}$ as a Cartier divisor and take closure in $X$.) Then $f^{*} \Delta_{A} \geq \Delta_{X}$ and so $m K_{X}+m f^{*} \Delta_{A} \geq f^{*}(k L)$.

(Proof of (2) of Theorem 1.3). By Proposition 9.2, we have

$$
\kappa\left(X, K_{X}\right) \geq \kappa\left(X, f^{*}\left((g+n+1) L-\Delta_{A}\right)\right) \geq \kappa\left(A(\Gamma)^{\Sigma},(g+n+1) L-\Delta_{A}\right) .
$$

On the other hand, since

$$
H^{0}\left(X, K_{X}^{\otimes m^{\prime}}\right) \subset H^{0}\left(X, K_{X}^{\otimes m^{\prime}}\left(m^{\prime} \Delta_{X}\right)\right) \simeq M_{m^{\prime}(g+n+1)}(\Gamma)
$$

for every $m^{\prime}$, we have $\kappa\left(X, K_{X}\right) \leq \kappa\left(A(\Gamma)^{\Sigma},(g+n+1) L\right)=g(g+1) / 2$.

\section{Singularities}

In this section, which is largely independent of the previous sections, we prove that $X^{n}(\Gamma)$ has canonical singularities in most cases (Proposition 10.3). Below, by a representation of a finite group $G$ over a field $K$, we mean a finite dimensional $K$-linear space equipped with a linear action of $G$. $(K$ will be either $\mathbb{Q}$ or $\mathbb{C}$.) We write $e(\alpha)=\exp (2 \pi i \alpha)$ for $\alpha \in \mathbb{Q} / \mathbb{Z}$.

Let $W$ be a representation of a finite group $G$ over $\mathbb{C}$. The Reid-ShepherdBarron-Tai criterion [24], [30] tells whether $W / G$ has canonical singularities in terms of the eigenvalues of elements of $G$. Let $\gamma \in G$ and $e\left(\alpha_{1}\right), \cdots, e\left(\alpha_{d}\right)$ be the eigenvalues of $\gamma$ on $W$ where $d=\operatorname{dim} W$. We choose $\alpha_{i} \in \mathbb{Q}$ from $0 \leq \alpha_{i}<1$. The Reid-Tai sum of $\gamma$ is defined by

$$
R T_{\gamma}(W)=\sum_{i=1}^{d} \alpha_{i}
$$

The action of $\gamma$ on $W$ is called quasi-reflection (or pseudo-reflection) if its eigenvalues are $1, \cdots, 1, \lambda$ with $\lambda \neq 1$.

Theorem 10.1 ([24], [30]). Assume that G contains no quasi-reflection on $W$. Then $W / G$ has canonical singularities if and only if $R T_{\gamma}(W) \geq 1$ for every element $\gamma \neq \mathrm{id}$ of $G$.

We will apply this RST criterion for $W$ the tangent space $T_{p} \mathcal{X}^{(n)}$ of $\mathcal{X}^{(n)}$ at a point $p \in \mathcal{X}^{(n)}$ and $G$ the stabilizer of $p$ in $\Gamma$.

10.1. Distribution of eigenvalues. We first prepare a lemma on the distribution of eigenvalues. Let $G=\mathbb{Z} / N$ be the standard cyclic group of order $N$. For $k \in \mathbb{Z} / N$ we write $\chi_{k / N}$ for the 1-dimensional $\mathbb{C}$-representation of $G$ on which the standard generator $\overline{1} \in G$ acts by $e(k / N)$. Recall $([26] \S 13.1)$ 
that there is a unique faithful $\mathbb{Q}$-representation $\mathbb{V}_{N}$ of $G$ that is irreducible over $\mathbb{Q}$. The complexification of $\mathbb{V}_{n}$ decomposes as

$$
V_{N}:=\mathbb{V}_{N} \otimes_{\mathbb{Q}} \mathbb{C} \simeq \bigoplus_{k \in(\mathbb{Z} / N)^{\times}} \chi_{k / N}
$$

For $d \mid N, \mathbb{V}_{d}$ is a representation of $G$ via the reduction $\mathbb{Z} / N \rightarrow \mathbb{Z} / d$. It is classical ([26] §13.1) that every $\mathbb{Q}$-representation of $G$ decomposes over $\mathbb{Q}$ into a direct sum of $\mathbb{V}_{d_{1}}, \cdots, \mathbb{V}_{d_{a}}$ for some $d_{1}, \cdots, d_{a} \mid N$. (We may have $d_{i}=d_{j}$ for $i \neq j$.)

Lemma 10.2. Let $\Lambda_{\mathbb{Q}}$ be a representation of $G$ over $\mathbb{Q}$ and

$$
\Lambda_{\mathbb{Q}}=\bigoplus_{i=1}^{a} \mathbb{V}_{d_{i}}
$$

be an irreducible decomposition of $\Lambda_{\mathbb{Q}}$ over $\mathbb{Q}$. Assume that $G$ preserves a weight 1 Hodge decomposition $\Lambda_{\mathbb{C}}=V \oplus \bar{V}$ of $\Lambda_{\mathbb{C}}$.

(1) Let $d>2$. If $\mathbb{V}_{d}^{\oplus k}$ appears in (10.1), there is a sub G-representation $W_{d}$ of $V$ such that $W_{d} \oplus \bar{W}_{d} \simeq V_{d}^{\oplus k}$ as representations of $G$ over $\mathbb{C}$.

(2) For $d=1,2$ the multiplicity of $\mathbb{V}_{d}$ in (10.1) is even, say $2 k$, and $V$ contains a sub G-representation $V^{\prime}$ isomorphic to $V_{d}^{\oplus k}$.

Proof. (1) Let $d>2$. For a C-representation $W$ of $G$ we write $\lambda(W)$ for the set of eigenvalues of $\overline{1} \in G$ counted with multiplicity. We choose eigendecompositions of $V$ and $\bar{V}$ with respect to $\overline{1} \in G$ :

$$
V=\bigoplus_{\lambda_{\alpha} \in \lambda(V)} \mathbb{C} v\left(\lambda_{\alpha}\right), \quad \bar{V}=\bigoplus_{\lambda_{\beta}^{\prime} \in \lambda(\bar{V})} \mathbb{C} w\left(\lambda_{\beta}^{\prime}\right),
$$

where $v\left(\lambda_{\alpha}\right) \in V$ is a $\lambda_{\alpha}$-eigenvector and $w\left(\lambda_{\beta}^{\prime}\right) \in \bar{V}$ a $\lambda_{\beta}^{\prime}$-eigenvector. We also fix a decomposition $\lambda\left(\Lambda_{\mathbb{C}}\right)=\lambda(V) \sqcup \lambda(\bar{V})$. Now, since $\Lambda_{\mathbb{Q}}$ contains $\mathbb{V}_{d}^{\oplus k}$, there exists some embedding

$$
\theta: \lambda\left(V_{d}^{\oplus k}\right) \hookrightarrow \lambda\left(\Lambda_{\mathbb{C}}\right)=\lambda(V) \sqcup \lambda(\bar{V}) .
$$

$\left(\mu \in \lambda\left(V_{d}^{\oplus k}\right)\right.$ and $\theta(\mu) \in \lambda\left(\Lambda_{\mathbb{C}}\right)$ are the same number.) We put the elements of $\lambda\left(V_{d}^{\oplus k}\right)$ by the order of their angle in $(0,2 \pi)$, say $\lambda\left(V_{d}^{\oplus k}\right)=\left\{\mu_{1}, \cdots, \mu_{l}\right\}$. Then $\mu_{l+1-i}=\bar{\mu}_{i}$, and $\mu_{i}$ has angle in $(0, \pi)$ if $i \leq l / 2$. We put

$$
W_{d}^{+}:=\bigoplus_{\substack{i \leq l / 2 \\ \theta\left(\mu_{i}\right) \in \lambda(V)}} \mathbb{C} v\left(\theta\left(\mu_{i}\right)\right), \quad W_{d}^{-}:=\bigoplus_{\substack{j \leq l / 2 \\ \theta\left(\mu_{j}\right) \in \lambda(\bar{V})}} \mathbb{C w ( \theta ( \mu _ { j } ) )} .
$$

Since $v\left(\lambda_{\alpha}\right) \in V$ and $w\left(\lambda_{\beta}^{\prime}\right) \in \bar{V}$, we have $W_{d}^{+}, W_{d}^{-} \subset V$. We also have $W_{d}^{+} \cap W_{d}^{-}=\{0\}$ because elements of $\lambda\left(W_{d}^{+}\right)$have angle in $(0, \pi)$ while those 
of $\lambda\left(W_{d}^{-}\right)$in $(\pi, 2 \pi)$. Then we put $W_{d}=W_{d}^{+} \oplus W_{d}^{-}$. Since $\lambda\left(W_{d}^{+}\right) \sqcup \lambda\left(\overline{W_{d}^{-}}\right)=$ $\left\{\mu_{1}, \cdots, \mu_{l / 2}\right\}$, by construction, we have

$$
\lambda\left(W_{d} \oplus \bar{W}_{d}\right)=\lambda\left(W_{d}^{+}\right) \sqcup \lambda\left(W_{d}^{-}\right) \sqcup \lambda\left(\overline{W_{d}^{+}}\right) \sqcup \lambda\left(\overline{W_{d}^{-}}\right)=\left\{\mu_{1}, \cdots, \mu_{l}\right\} .
$$

Therefore $W_{d} \oplus \bar{W}_{d} \simeq V_{d}^{\oplus k}$ as abstract $G$-representations.

(2) Let $d=1$ or 2 . Let $\mathbb{W} \subset \Lambda_{\mathbb{Q}}$ be the direct sum of all components $\mathbb{V}_{d_{i}}$ in (10.1) such that $d_{i}=d$. Then $W=\mathbb{W} \otimes_{\mathbb{Q}} \mathbb{R}$ is the $( \pm 1)$-eigenspace of $\overline{1} \in G$ on $\Lambda_{\mathbb{R}}$. Let $J: \Lambda_{\mathbb{R}} \rightarrow \Lambda_{\mathbb{R}}$ be the complex structure given by the Hodge decomposition $\Lambda_{\mathbb{C}}=V \oplus \bar{V}$. Since the $G$-action commutes with $J, J$ preserves $W$ and gives a complex structure on $W$. In particular, $\mathbb{W}$ has even dimension. If $W_{\mathbb{C}}=V^{\prime} \oplus \bar{V}^{\prime}$ is the Hodge decomposition given by $\left.J\right|_{W}$, then $V^{\prime}=V \cap W_{\mathbb{C}}$ is the $( \pm 1)$-eigenspace of $\overline{1} \in G$ on $V$.

10.2. Singularities of $X^{n}(\Gamma)$. As before, let $\Lambda$ be a symplectic lattice of rank $2 g>2$ and $\Gamma$ a finite-index subgroup of $\operatorname{Sp}(\Lambda)$. Our main result of $\$ 10$ is the following.

Proposition 10.3. The Kuga variety $X^{n}(\Gamma)$ has canonical singularities unless when $(g, n)=(2,1),(3,1),(2,2)$ and $\Gamma$ contains an element of order 6 whose eigenvalues on $\Lambda_{\mathbb{C}}$ are $e(1 / 6), e(-1 / 6), 1, \cdots, 1$.

Proof. Recall from $\$ 2.2$ that $X^{n}(\Gamma)=\mathcal{X}^{(n)} / \Gamma$. Let $p=\left([V], x_{1}, \cdots, x_{n}\right)$ be a point of $\mathcal{X}^{(n)}$ where $[V] \in \mathcal{D}$ and $x_{i} \in V^{\vee} / \Lambda$. It suffices to show that $T_{p} \mathcal{X}^{(n)} / \Gamma_{p}$ has canonical singularities where $\Gamma_{p}<\Gamma$ is the stabilizer of $p$. By Lemma 7.1, $\Gamma_{p}$ contains no quasi-reflection on $T_{p} \mathcal{X}^{(n)}$. Thus it suffices to show that $R T_{\gamma}\left(T_{p} \mathcal{X}^{(n)}\right) \geq 1$ for every $\gamma \neq \mathrm{id} \in \Gamma_{p}$. Since

$$
T_{p} \mathcal{X}^{(n)} \simeq T_{x_{1}}\left(V^{\vee} / \Lambda\right) \oplus \cdots \oplus T_{x_{n}}\left(V^{\vee} / \Lambda\right) \oplus T_{[V]} \mathcal{D} \simeq\left(V^{\vee}\right)^{\oplus n} \oplus \mathrm{Sym}^{2} V^{\vee}
$$

as $\Gamma_{p}$-representations, we are reduced to the following calculation in linear algebra. (We rewrite $V^{\vee}$ as $V$, and $\Lambda_{\mathbb{Q}}^{\vee}$ as $\Lambda_{\mathbb{Q}}$.)

Lemma 10.4. Let $G=\langle\gamma\rangle$ be a finite cyclic group and $\Lambda_{\mathbb{Q}}$ a representation of $G$ over $\mathbb{Q}$ of dimension $2 g>2$. Assume that $G$ preserves a Hodge decomposition $\Lambda_{\mathbb{C}}=V \oplus \bar{V}$ and that $\left(n, \Lambda_{\mathbb{Q}}\right)$ is neither of the following:

- $n=1, \Lambda_{\mathbb{Q}} \simeq \mathbb{V}_{6} \oplus \mathbb{V}_{1}^{\oplus 2 g-2}$ with $g=2,3$.

- $n=2, \Lambda_{\mathbb{Q}} \simeq \mathbb{V}_{6} \oplus \mathbb{V}_{1}^{\oplus 2}(g=2)$.

Then $R T_{\gamma}\left(V^{\oplus n} \oplus \operatorname{Sym}^{2} V\right) \geq 1$.

Proof. As $G$-representation, one of the following cases occur:

(1) $\Lambda_{\mathbb{Q}} \supset \mathbb{V}_{d}, \varphi(d)>2$;

(2) $\Lambda_{\mathbb{Q}} \supset \mathbb{V}_{3}$;

(3) $\Lambda_{\mathbb{Q}} \supset \mathbb{V}_{4}$;

(4) $\Lambda_{\mathbb{Q}} \supset \mathbb{V}_{6}$;

(5) $\Lambda_{\mathbb{Q}}=\mathbb{V}_{1}^{\oplus 2 k} \oplus \mathbb{V}_{2}^{\oplus 2 l}$. 
We estimate the Reid-Tai sum case-by-case. As in the proof of Lemma10.1, for a $G$-representation $W$ over $\mathbb{C}$, we write $\lambda(W)$ for the set of eigenvalues of $\gamma$ counted with multiplicity. By associating $\alpha \in[0,1)$ to $e(\alpha)$, we identify elements of $\lambda(W)$ with rational numbers in $[0,1)$. We write $S^{2} V=\operatorname{Sym}^{2} V$.

We first consider the case $n=1$. We write $R T=R T_{\gamma}\left(V \oplus S^{2} V\right)$. We show that $R T \geq 1$ unless $\Lambda_{\mathbb{Q}} \simeq \mathbb{V}_{6} \oplus \mathbb{V}_{1}^{\oplus 2 g-2}$ with $g \leq 3$.

(1) Let $W_{d} \subset V$ be the sub $G$-representation such that $W_{d} \oplus \bar{W}_{d} \simeq V_{d}$ as constructed in Lemma 10.2. Firstly, if $\lambda\left(W_{d}\right)$ contains two elements $\lambda, \lambda^{\prime}$ from $(1 / 2,1)$, we have $R T>\lambda+\lambda^{\prime}>1$. Secondly, suppose that $\lambda\left(W_{d}\right)$ contains exactly one element $\lambda$ from $(1 / 2,1)$. Since $W_{d} \oplus \bar{W}_{d} \simeq V_{d}$, every element of $\lambda\left(V_{d}\right) \cap(0,1 / 2)$ except $1-\lambda$ appears in $\lambda\left(W_{d}\right)$. Let $\lambda^{\prime}$ be the maximal element of $\lambda\left(W_{d}\right) \cap(0,1 / 2)$. When $\lambda^{\prime}>1-\lambda$, we have $R T \geq$ $\lambda+\lambda^{\prime}>1$. When $\lambda^{\prime}<1-\lambda$, we have $\lambda+\lambda^{\prime} \in \lambda\left(S^{2} V\right)$ and $\lambda+\lambda^{\prime}<1$. Then $R T \geq \lambda+\left(\lambda+\lambda^{\prime}\right)>2 \lambda>1$. Thirdly, if all elements of $\lambda\left(W_{d}\right)$ are contained in $(0,1 / 2)$, we have $\lambda\left(W_{d}\right)=\lambda\left(V_{d}\right) \cap(0,1 / 2)$ by $V_{d} \simeq W_{d} \oplus \bar{W}_{d}$. Let $\lambda$ be the maximal element of $\lambda\left(W_{d}\right)$. Then $\lambda>1 / 4$. Since $\lambda\left(S^{2} V\right)$ contains $\lambda+\lambda^{\prime}<1$ for every $\lambda^{\prime} \in \lambda(V)$, we have $R T>(2+\varphi(d) / 2) \lambda \geq 4 \lambda>1$.

(2) By Lemma 10.2, either $\chi_{1 / 3} \subset V$ or $\chi_{2 / 3} \subset V$. In the first case, we have $1 / 3 \in \lambda(V)$ and $2 / 3 \in \lambda\left(S^{2} V\right)$, so $R T \geq 1 / 3+2 / 3=1$. In the second case, we have $2 / 3 \in \lambda(V)$ and $1 / 3 \in \lambda\left(S^{2} V\right)$, so again $R T \geq 1$.

(3) Since $g>1$, we have $\Lambda_{\mathbb{Q}} \neq \mathbb{V}_{4}$. In view of the cases (1), (2), we only need to consider the case $\mathbb{V}_{4} \oplus \mathbb{V}_{d} \subset \Lambda_{\mathbb{Q}}$ with $d=1,2,4,6$. When $\mathbb{V}_{4}^{\oplus 2} \subset \Lambda_{\mathbb{Q}}$, $\lambda(V)$ contains two elements from $\{1 / 4,1 / 4,3 / 4,3 / 4\}$ by Lemma 10.2, so $\lambda\left(S^{2} V\right)$ contains two $1 / 2$ and hence $R T>1$. When $\mathbb{V}_{4} \oplus \mathbb{V}_{6} \subset \Lambda_{\mathbb{Q}}, \lambda(V)$ contains $\{1 / 4$ or $3 / 4,1 / 6$ or $5 / 6\}$ by Lemma 10.2. Then $\lambda\left(S^{2} V\right)$ contains $\{1 / 2,1 / 3$ or $2 / 3\}$. Hence $R T>1$. Similarly, when $\mathbb{V}_{4} \oplus \mathbb{V}_{2} \subset \Lambda_{\mathbb{Q}}, \lambda(V)$ contains $\{1 / 2,1 / 4$ or $3 / 4\}$, and so $\lambda\left(S^{2} V\right)$ contains $\{3 / 4$ or $1 / 4,1 / 2\}$, which implies $R T>1$. Finally, when $\mathbb{V}_{4} \oplus \mathbb{V}_{1} \subset \Lambda_{\mathbb{Q}}, \lambda(V)$ contains $\{0,1 / 4$ or $3 / 4\}$, and so $\lambda\left(S^{2} V\right)$ contains $\{1 / 2,1 / 4$ or $3 / 4\}$. This proves $R T \geq 1$.

(4) In view of the cases (1) - (3), we only need to cover the cases

$$
\Lambda_{\mathbb{Q}} \supset \mathbb{V}_{6} \oplus \mathbb{V}_{6}, \quad \Lambda_{\mathbb{Q}} \supset \mathbb{V}_{6} \oplus \mathbb{V}_{2}, \quad \Lambda_{\mathbb{Q}}=\mathbb{V}_{6} \oplus \mathbb{V}_{1}^{\oplus 2 g-2}(g \geq 4) .
$$

When $\mathbb{V}_{6}^{\oplus 2} \subset \Lambda_{\mathbb{Q}}, \lambda(V)$ contains two elements from $\{1 / 6,1 / 6,5 / 6,5 / 6\}$ by Lemma10.2, so $\lambda\left(S^{2} V\right)$ contains two elements from $\{1 / 3,1 / 3,2 / 3,2 / 3\}$. It follows that $R T \geq 1$. When $\mathbb{V}_{6} \oplus \mathbb{V}_{2} \subset \Lambda_{\mathbb{Q}}, \lambda(V)$ contains $\{1 / 2,1 / 6$ or $5 / 6\}$, so $\lambda\left(S^{2} V\right)$ contains $1 / 3$ or $2 / 3$. Thus $R T \geq 1$. Finally, when $\Lambda_{\mathbb{Q}}=\mathbb{V}_{6} \oplus$ $\mathbb{V}_{1}^{\oplus 2 g-2}$, we have $V \simeq \chi_{ \pm 1 / 6} \oplus \chi_{1}^{\oplus g-1}$. If $V \simeq \chi_{5 / 6} \oplus \chi_{1}^{\oplus g-1}$, then $\chi_{5 / 6} \subset S^{2} V$, and so $R T>1$. If $V \simeq \chi_{1 / 6} \oplus \chi_{1}^{\oplus g-1}$, we have $R T=1 / 3+g / 6 \geq 1$ by $g \geq 4$.

(5) In this case we have $V \simeq \chi_{1}^{\oplus k} \oplus \chi_{1 / 2}^{\oplus l}$ by Lemma 10.2. When $l \geq 2$, we have $R T \geq l / 2 \geq 1$. When $l=1$, we have $k \geq 1$ by $g \geq 2$. Then $1 / 2 \in \lambda(V)$ and $1 / 2 \in \lambda\left(S^{2} V\right)$, so $R T \geq 1$. This finishes the proof for the case $n=1$. 
Next let $n \geq 2$. Since $R T_{\gamma}\left(V^{\oplus n} \oplus S^{2} V\right) \geq R T_{\gamma}\left(V \oplus S^{2} V\right)$, we only need to consider the case $\Lambda_{\mathbb{Q}}=\mathbb{V}_{6} \oplus \mathbb{V}_{1}^{\oplus 2 g-2}$ and $V \simeq \chi_{1 / 6} \oplus \chi_{1}^{\oplus g-1}$ with $g=2,3$ by the above proof for the case $n=1$. In this case the Reid-Tai sum is $1 / 3+(g+n-1) / 6$, which is smaller than 1 only when $(g, n)=(2,2)$. This completes the proof of Lemma 10.4 and hence of Proposition 10.3 .

\section{REFERENCES}

[1] Ash, A.; Mumford, D.; Rapoport, M.; Tai, Y. Smooth compactifications of locally symmetric varieties. 2nd ed. Cambridge. Univ. Press (2010).

[2] Baily, W. L., Jr.; Borel, A. Compactification of arithmetic quotients of bounded symmetric domains. Ann. of Math. (2) 84 (1966), 442-528.

[3] Faltings, G.; Chai, C. Degeneration of abelian varieties. Springer, 1990.

[4] Farkas, G.; Verra, A. The universal abelian variety over $A_{5}$. Ann. Sci. Éc. Norm. Supér. (4) 49 (2016), no. 3, 521-542.

[5] Freitag, E. Siegelsche Modulfunktionen. Springer, 1983.

[6] Gunji, K. Defining equations of the universal abelian surfaces with level three structure. Manuscripta Math. 119 (2006), no. 1, 61-96.

[7] Hartshorne, R. Algebraic Geometry. GTM 52, Springer, 1977.

[8] Hatada, K. Siegel cusp forms as holomorphic differential forms on certain compact varieties. Math. Ann. 262 (1983), no. 4, 503-509.

[9] Hulek, K.; Kahn, C.; Weintraub, S. Moduli spaces of abelian surfaces: compactification, degenerations, and theta functions. de Gruyter, 1993.

[10] Igusa, J. On Siegel modular forms of genus two. Amer. J. Math. 84 (1962), 175-200.

[11] Igusa, J. On the irreducibility of Schottky's divisor. J. Fac. Sci. Univ. Tokyo 28 (1982), 531-545.

[12] Iitaka, S. Algebraic geometry. GTM 76, Springer, 1982.

[13] Kollár, J; Mori, S. Birational geometry of algebraic varieties. Cambridge Univ. Press, 1998.

[14] Lan, K. Toroidal compactifications of PEL-type Kuga families. Algebra Number Theory 6 (2012), no. 5, 885-966.

[15] Looijenga, E. Moduli spaces and locally symmetric varieties. in "Development of moduli theory - Kyoto 2013”, 33-75, Adv. Stud. Pure Math., 69, MSJ, 2016.

[16] Ma, S. Modular forms of weight $3 m$ and elliptic modular surfaces. Proc. Japan Acad. Ser. A 95 (2019), no. 4, 31-36.

[17] Mumford, D. Hirzebruch's proportionality theorem in the noncompact case. Invent. Math. 42 (1977), 239-272.

[18] Mumford, D.; Fogarty, J.; Kirwan, F. Geometric invariant theory. Springer, 1994.

[19] Nakayama, N. Zariski-decomposition and abundance. MSJ Memoirs 14, 2004.

[20] Namikawa, Y. A new compactification of the Siegel space and degeneration of Abelian varieties. I, II. Math. Ann. 221 (1976), 97-141, 201-241.

[21] Nebe, G.; Venkov, B. On Siegel modular forms of weight 12. J. Reine Angew. Math. 531 (2001), 49-60.

[22] Pink, R. Arithmetic compactification of mixed Shimura varieties. Thesis, Rheinischen Friedrich-Wilhelms-Universität, Bonn, 1989.

[23] Poor, C.; Yuen, D. S. Computations of spaces of Siegel modular cusp forms. J. Math. Soc. Japan 59 (2007), no. 1, 185-222. 
[24] Reid, M. Canonical 3-folds. in "Journées de Géometrie Algébrique d'Angers", 273310, Sijthoff \& Noordhoff, 1980.

[25] Salvati-Manni, R. Modular forms of the fourth degree. in "Classification of irregular varieties (Trento, 1990)", 106-111, Lecture Notes in Math., 1515, Springer, 1992.

[26] Serre, J.-P. Linear representations of finite groups. GTM 42, Springer, 1977.

[27] Shimura, G. Moduli and fibre systems of abelian varieties. Ann. of Math. (2) 83 (1966), 294-338.

[28] Shioda, T. On elliptic modular surfaces. J. Math. Soc. Japan 24 (1972), 20-59.

[29] Shokurov, V. V. Holomorphic differential forms of higher degree on Kuga's modular varieties. Math. USSR-Sb., 30 (1976), no. 1, 119-142.

[30] Tai, Y.-S. On the Kodaira dimension of the moduli space of abelian varieties. Invent. Math. 68 (1982), no. 3, 425-439.

[31] Tsuyumine, S. On Siegel modular forms of degree three. Amer. J. Math. 108 (1986), no. 4, 755-862, 1001-1003.

[32] Ueno, K. On algebraic fibre spaces of abelian varieties. Math. Ann. 237 (1978), no. $1,1-22$.

[33] Verra, A. On the universal principally polarized abelian variety of dimension 4. in "Curves and abelian varieties", 253-274, Contemp. Math., 465, AMS, 2008.

Department of Mathematics, Tokyo Institute of Technology, Tokyo 152-8551, JaPan

E-mail address: ma@math.titech.ac.jp 